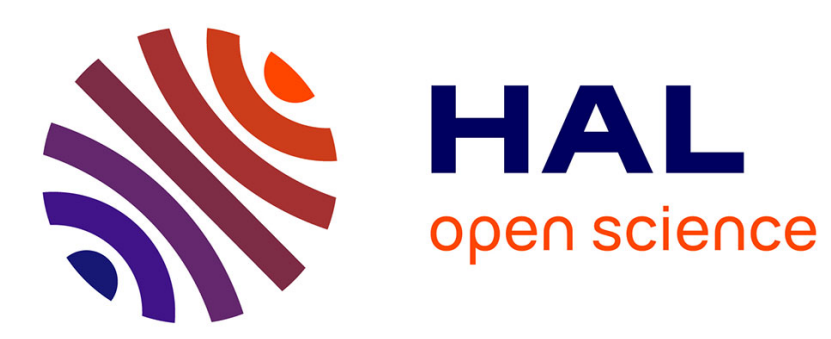

\title{
Magnetic Anisotropy in a Cubane-like Ni_4 Complex: An Ab Initio Perspective
}

Rémi Maurice

\section{To cite this version:}

Rémi Maurice. Magnetic Anisotropy in a Cubane-like Ni_4 Complex: An Ab Initio Perspective. Inorganic Chemistry, 2021, 60 (9), pp.6306-6318. 10.1021/acs.inorgchem.1c00047 . hal-03217634

\section{HAL Id: hal-03217634 \\ https://hal.science/hal-03217634}

Submitted on 8 Oct 2021

HAL is a multi-disciplinary open access archive for the deposit and dissemination of scientific research documents, whether they are published or not. The documents may come from teaching and research institutions in France or abroad, or from public or private research centers.
L'archive ouverte pluridisciplinaire HAL, est destinée au dépôt et à la diffusion de documents scientifiques de niveau recherche, publiés ou non, émanant des établissements d'enseignement et de recherche français ou étrangers, des laboratoires publics ou privés. 


\title{
Magnetic anisotropy in a cubane-like $\mathbf{N i}_{4}$ Complex: An ab initio perspective
}

\author{
Rémi Maurice* \\ SUBATECH, UMR CNRS 6457, IN2P3/IMT Atlantique/Université de Nantes, 4 rue A. \\ Kastler, 44307 Nantes Cedex 3, France \\ E-mail: remi.maurice@subatech.in2p3.fr
}


Prepared for Inorg. Chem.

Date of revision: March 9, 2021.

\begin{abstract}
Magnetic anisotropy, in the absence of an external magnetic field, relates to the degeneracy lift of energy levels. In the standard case of transition metal complexes, this property is usually modelled by an anisotropic spin Hamiltonian and one speaks of 'zero-field splitting' (ZFS) of spin states. While the case of mononuclear complexes has been extensively described by means of $a b$ initio quantum mechanical calculations, the literature on polynuclear complexes studied with these methodologies is rather scarse. In this work, advanced multiconfigurational wave function theory methods are applied to compute the ZFS of the ground $S=4$ state of an actual tetranickel(II) complex, displaying a magnet behavior below $0.5 \mathrm{~K}$. First, the isotropic couplings are computed in the absence of the spin-orbit coupling operator, in the full complex and also in clusters with only two active nickel(II) centers, confirming the occurence of weak ferromagnetic couplings in this system. Second, the single-site magnetic anisotropies are computed on a cluster bearing only one active nickel(II) site, showing that the single-site anisotropy axes are not oriented in an optimal fashion for generating a large uniaxial molecular anisotropy. Furthermore, the possibility for involving only a few local orbital excited states in the calculation is assessed, actually opening the way for a consistent and manageable treatment of the ZFS of the ground $S=4$ state. Third, multiconfigurational calculations are performed on the full complex, confirming the weak uniaxial anisotropy occuring for this state, and also, interestingly, revealing a significant contribution of the lowest-lying orbitally excited $S=3$ states. Overall, by comparison with experiment, the reported results question the common habit of using only one structure, in particular derived from a crystallography experiment, to compute magnetic anisotropy parameters.
\end{abstract}




\section{Introduction}

Many single-molecule magnets (SMMs) have been proposed since the discovery of the intriguing properties of the now famous $\mathrm{Mn}_{12}$ complex. ${ }^{1-4}$ Several strategies for improving the blocking temperatures have been envisaged, as (i) attempting to reach the largest possible molecular spin state, which is somehow vain ${ }^{5}$ to enlarge $-D S^{2}$ (the "energy barrier" for even $S$ cases), (ii) playing with the local coordination environment of the paramagnetic ions to enlarge the single-site anisotropies, ${ }^{6}$ (iii) playing with the orientation of the single-site anisotropy axes ${ }^{7}$ (iv) triggering "first-order" spin-orbit coupling (SOC) situations, as it is the case for instance for lanthanide $\mathrm{SMMs}^{8}$ or $(\mathrm{v})$ limit the relaxation mechanisms notably by reducing the spin-phonon coupling. ${ }^{9}$ So far, the highest reported blocking temperatures concern dysprosium complexes, ${ }^{10-13}$ with a current record at $80 \mathrm{~K} .{ }^{13}$

In the field of molecular magnetism, large polynuclear SMMs have been modelled quantum mechanically by density functional theory (DFT), following the pioneering work of Pederson and Khanna. ${ }^{14}$ Many polynuclear transition metal complexes have been successfully treated, ${ }^{15,16}$ with usually a very good agreement between the computed and experimental axial ZFS parameter, $D$, which at least in first intention, is key to describe the "spin-based" SMMs (by opposition to the "SOC-based" ones, whose properties cannot be fully modeled by simple spin Hamiltonians). However, this methodology somehow fails for describing mononuclear nickel(II) complexes, ${ }^{17}$ for reasons which remain to be better elucidated, which may question its consistent applicability to polynuclear nickel(II) complexes.

Among the polynuclear nickel(II) complexes, several cubane-like structures have been reported in the literature. ${ }^{18-21}$ Typical features include weak isotropic couplings and small uniaxial molecular anisotropies. In this work, the complex reported by Moragues-Cánovas et al. has been arbitrarily selected as a case study to showcase the capabilities and challenges that arise from the wave function theory (WFT) framework (note that it has previously been the subject of a DFT study devoted to the isotropic couplings, ${ }^{22}$ as also for another

nickel(II) cubane-like complex $\left.{ }^{23,24}\right)$. In contrast with DFT, multiconfigurational and rela- 
tivistic wave function calculations have become a standard to compute the ZFS in mononuclear complexes, ${ }^{25-29}$ with notably many successful applications on mononuclear nickel(II) ones. $^{17,30-41}$

The actual description of the magnetic anisotropy in binuclear nickel(II) complexes is limited to a few papers. ${ }^{7,42,43}$ These studies have reached several conclusions that are of interest for the present paper. First, the mapping between the multispin and the giant spin Hamiltonians is somehow intractable if one uses a multispin Hamiltonian that involves a biquadratic anisotropic exchange term. ${ }^{42,43}$ Consequently, a cluster approach consisting of computing single-site and inter-site isotropic and anisotropic terms is not a practical way of deriving the giant spin Hamiltonian for the ground $S=4$ state of the complex of interest. Therefore, this ZFS will be computed with the four active nickel(II) sites, which, as we will see, appears to be actually very demanding. Second, the computation of the single-site anisotropic parameters can be done with only one active nickel(II) site. ${ }^{7}$ Third, knowledge of the orientations of the single-site anisotropy axes may reveal potential synergies or interferences between the single-site anisotropies. ${ }^{7}$ Note that concerning the comparison of experimental and computational giant spin Hamiltonian parameter values, the experimental $D$ value for the $S=2$ state of the $\left[\mathrm{Ni}_{2}(\mathrm{en})_{4} \mathrm{Cl}_{2}\right]^{2+}$ (en = ethylenediamine) complex, ${ }^{44}-3.0 \mathrm{~cm}^{-1}$, was fairly reproduced by the $-1.8 \mathrm{~cm}^{-1}$ computational one, ${ }^{43}$ as mentioned elsewhere. ${ }^{29}$

The paper is organised as follows. First, a theoretical framework will be given by introducing the structure and its symmetry properties, the Heisenberg-Dirac-van Vleck (HDVV) Hamiltonian and its topology for this system, the single-site excited states and their role on the single-site anisotropies, the giant spin Hamiltonian for the ground $S=4$ state and the used quantum mechanical methodologies as well as the associated computational details. Second, the results will be presented in three parts, corresponding the the previous items (i.e. the isotropic couplings, the single-site anisotropies and the giant spin Hamiltonian). Third, a discussion on the physics of the system and on the followed modelling practice will be done, before opening toward perspectives for the molecular magnetism community. 


\section{Theory and Methods}

\subsection{Preliminaries: Structure and symmetry of the cubane-like $\mathrm{Ni}_{4}$ complex}

The structure reported by Moragues-Cánovas et al. ${ }^{19}$ is considered in this work. The complex is characterized by a molecular charge of +4 , overall compensated by a mixture of $\mathrm{Na}^{+}$and $\mathrm{NO}_{3}^{-}$ions in the crystal, which are here neglected (only the complex is treated). The complex structure displays an $\mathrm{S}_{4}$ symmetry, meaning that the four nickel(II) ions are symmetry equivalent. In Figure $1, \mathrm{Ni}_{1}$ and $\mathrm{Ni}_{3}$ as well as $\mathrm{Ni}_{2}$ and $\mathrm{Ni}_{4}$ (and reciproqually) can exchanged by applying the $\mathrm{C}_{2}$ symmetry operation, while the other exchanges require the application of an $\mathrm{S}_{4}^{1}$ or $\mathrm{S}_{4}^{3}$ improper rotation. By symmetry, only two Ni-Ni distances apply: $3.057 \AA$ $\left(\mathrm{Ni}_{1}-\mathrm{Ni}_{3}\right.$ and $\left.\mathrm{Ni}_{2}-\mathrm{Ni}_{4}\right)$ and $3.067 \AA\left(\mathrm{Ni}_{1}-\mathrm{Ni}_{2}, \mathrm{Ni}_{1}-\mathrm{Ni}_{4}, \mathrm{Ni}_{2}-\mathrm{Ni}_{3}\right.$ and $\left.\mathrm{Ni}_{3}-\mathrm{Ni}_{4}\right)$.
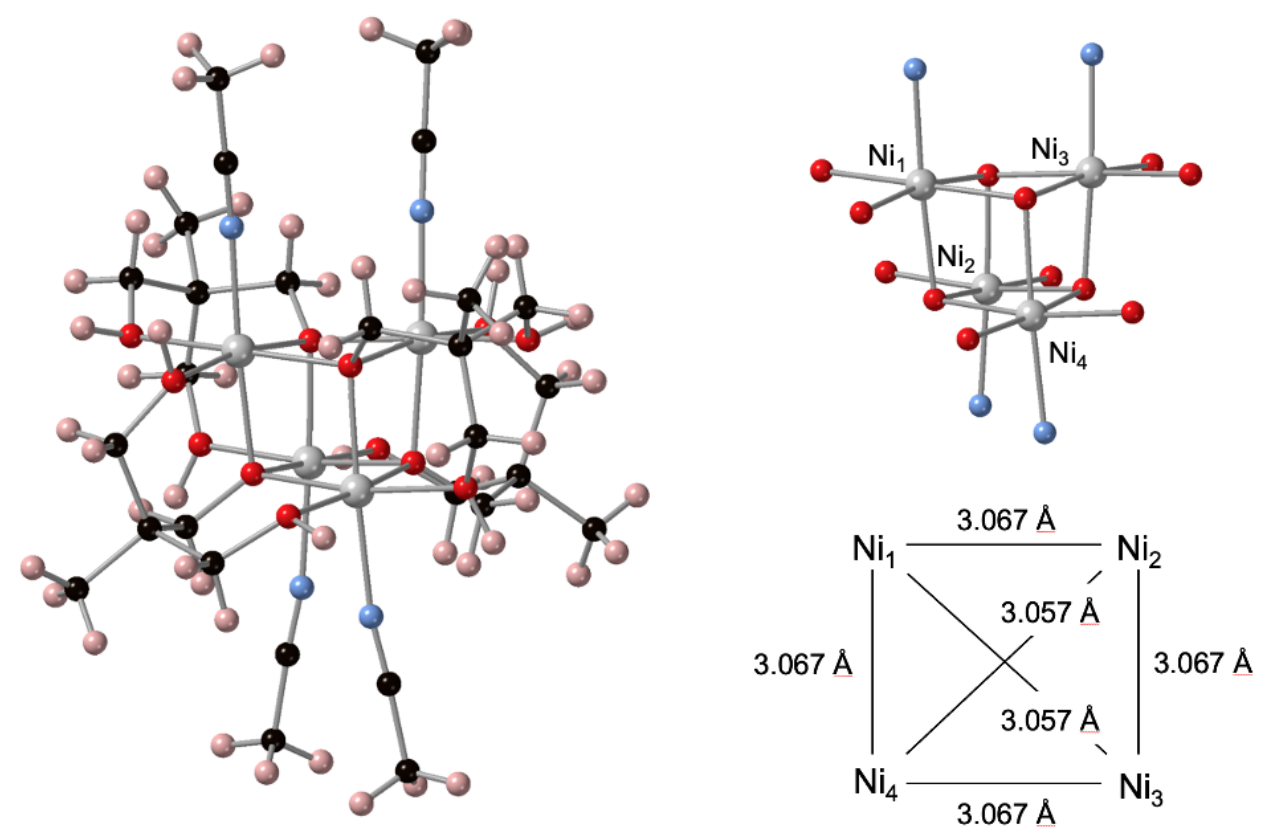

Figure 1: Ball and stick representations of the molecular structure of the studied cubane-like $\mathrm{Ni}_{4}$ complex, derived from the crystallographic one: ${ }^{19}$ all atoms (left); only the $\mathrm{Ni}, \mathrm{O}$ and $\mathrm{N}$ atoms (right). The $\mathrm{C}_{2}$ symmetry axis is vertical in the paper plane. Color code: gray stands for $\mathrm{Ni}$, red for $\mathrm{O}$, blue for $\mathrm{N}$ and pink for $\mathrm{H}$. A scheme recalls the $\mathrm{Ni}-\mathrm{Ni}$ distances. 
The coordination environment of the nickel(II) ions consists of distorted octahedra. Six distinct bond distances appear for the first coordination sphere atoms: the $\mathrm{Ni}-\mathrm{O}$ distances range between 2.023 and $2.051 \AA$ for the three cubane core oxygen atoms and are 2.054 and $2.056 \AA$ for the tripodal ligand oxygen atoms, while the $\mathrm{Ni-N}$ distance is $2.060 \AA$. It is important to stress here that all the $\measuredangle \mathrm{X}_{i}-\mathrm{Ni}-\mathrm{X}_{j}$ bond angles, $\mathrm{X}_{i}$ and $\mathrm{X}_{j}$ being neighbors in the first coordination sphere of the nickel(II) ion, deviate from the $90^{\circ}$ value. Finally, the three $\measuredangle \mathrm{Ni}_{i}-\mathrm{O}-\mathrm{Ni}_{j}$ bond angles for the cubane core oxygen atoms have pretty close values, actually ranging from 97.04 to $97.95^{\circ}$.

As stated by Venegas-Yazigi et al. in their previous DFT study, ${ }^{22}$ the crystal structure misses eight hydrogen atoms, located at the tripodal ligand terminations. In a similar way as in their study, the positions of these eight hydrogen atoms have been optimized. To do so, these positions have been initially defined so as to respect the $\mathrm{S}_{4}$ symmetry, relaxed in a blackbox way with the CrystalMaker software ${ }^{45}$ and slightly corrected after optimization to force the $\mathrm{S}_{4}$ symmetry. The resulting structure is given as Supporting Information. Note that the positions of terminating hydrogen atoms may affect the computed anisotropy parameters, as was observed in a dicobalt(II) system by Petit et al., ${ }^{46}$ and that this will be further commented in the Discussion Section.

\subsection{The Heisenberg-Dirac-van Vleck Hamiltonian, its topology and its solutions}

Before introducing model Hamiltonians for describing the spin anistropy, it is worth starting by introducing the Heisenberg-Dirac-van Vleck (HDVV) Hamiltonian. ${ }^{47-49}$ This Hamiltonian models the degeneracy lifts of spin states that essentially bear the same orbital wave functions. A typical situation for applying this Hamiltonian concerns polynuclear transition metal complexes, for which unpaired electrons are localized on distinct magnetic sites. Various formulae appear in the literature, depending on the prefactor that is applied together with the isotropic coupling parameters. In this work, the following expression is considered: 


$$
\hat{\mathbf{H}}^{\mathrm{HDVV}}=\sum_{<i, j>} J_{i j} \hat{\mathbf{S}}_{i} \cdot \hat{\mathbf{S}}_{j}
$$

where $J_{i j}$ is the isotropic coupling between two distinct $i$ and $j$ sites, $\hat{\mathbf{S}}_{i}$ and $\hat{\mathbf{S}}_{j}$ are the spin operator vectors on these sites, and the summation runs over all the independent pairs of magnetic sites.

Basically, two main routes from electronic structure theory calculations may be employed, ${ }^{50}$ (i) derive the couplings from spin-broken-symmetry calculations (spin unrestricted approach) or (ii) derive the couplings from multiconfigurational calculations that ensures the spin symmetry of the computed states (spin adapted approach). In the latter approach, followed in this work, the entire "Heisenberg spectrum" may be computed and deviations to the Landé intervals may reveal true physics ${ }^{51-54}$ and/or artifacts of the used computational approach. ${ }^{55,56}$ The choice of the orbitals to compute the states belonging to the Heisenberg space may be critical and it may be cleaner to compute them with a common set of orbitals (in particular within the state-average framework). ${ }^{50,56,57}$ The physics of the coupling has been the subject of many publications, among which one may quote a series of articles by Calzado et al. ${ }^{58-60}$ Naturally, many other articles have been key to understand this physics, as decribed for instance in the extensive review of Malrieu et al. ${ }^{61}$

As already mentioned, the complex under study displays an $\mathrm{S}_{4}$ symmetry. Thus, the number of free coupling parameters is drastically reduced by symmetry, and the HDVV Hamiltonian may be rewritten as follows:

$$
\hat{\mathbf{H}}^{\mathrm{HDVV}}=J_{1}\left(\hat{\mathbf{S}}_{1} \cdot \hat{\mathbf{S}}_{2}+\hat{\mathbf{S}}_{1} \cdot \hat{\mathbf{S}}_{4}+\hat{\mathbf{S}}_{2} \cdot \hat{\mathbf{S}}_{3}+\hat{\mathbf{S}}_{3} \cdot \hat{\mathbf{S}}_{4}\right)+J_{2}\left(\hat{\mathbf{S}}_{1} \cdot \hat{\mathbf{S}}_{3}+\hat{\mathbf{S}}_{2} \cdot \hat{\mathbf{S}}_{4}\right)
$$

where $J_{1}$ and $J_{2}$ correspond to the longer and shorter $\mathrm{Ni}-\mathrm{Ni}$ distances of the cubane core, respectively. Schematically, the topology of the isotropic couplings may be represented as in Figure 2 .

With four $S=1$ sites, the complex under study already displays a large manifold of 


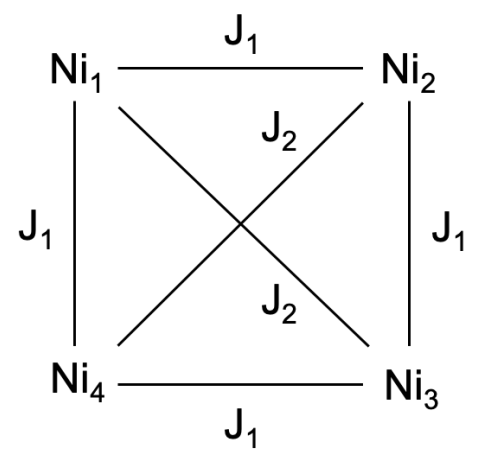

Figure 2: Topology of the isotropic couplings for the ground orbital configuration (adapted from Reference 18). Only two coupling constants apply ( $S_{4}$ symmetry).

states. Since the HDVV Hamiltonian naturally applies to the uncoupled basis ${ }^{62}$ i.e. to the $\left|S_{1}, M_{S 1}, S_{2}, M_{S 2}, S_{3}, M_{S 3}, S_{4}, M_{S 4}\right\rangle$ functions, one can easily determine its size: $3^{4}=81$. A convenient way of determining all the generated spin states consists in building a "spin tree" by successive coupling of the sites. When two $S_{i}$ and $S_{j}$ states are coupled, one may generate coupled $S_{i j}$ states that range between $\left|S_{i}-S_{j}\right|$ and $S_{i}+S_{j}$. For instance, the coupling of

two $S=1$ states generate three spin states with $S=0,1$ and 2 , respectively. Continuing the process up to four coupled $S=1$ states leads to one $S=4$, three $S=3$, six $S=2$, six $S=1$ and three $S=0$ spin states (see Figure 3 ).

Due to the $\mathrm{S}_{4}$ symmetry, some solutions of the HDVV Hamiltonian are actually doubly degenerate. The solutions corresponding to this situation are actually available for instance in the work of Escuer et al. ${ }^{18}$ Note that these solutions need to be adapted to fit the present Hamiltonian and labels: $J_{1}$ and $J_{2}$ need to be inverted and all the energies must be multiplied by a -2 factor. The resulting solutions are given in Table 1 .

\subsection{The lowest-lying single-site excited states and the single-site anisotropies}

Various strategies can be envisaged to compute single-site anisotropies in a polynuclear complex, (i) replace all the paramagnetic ions but one with a diamagnetic ion of similar 


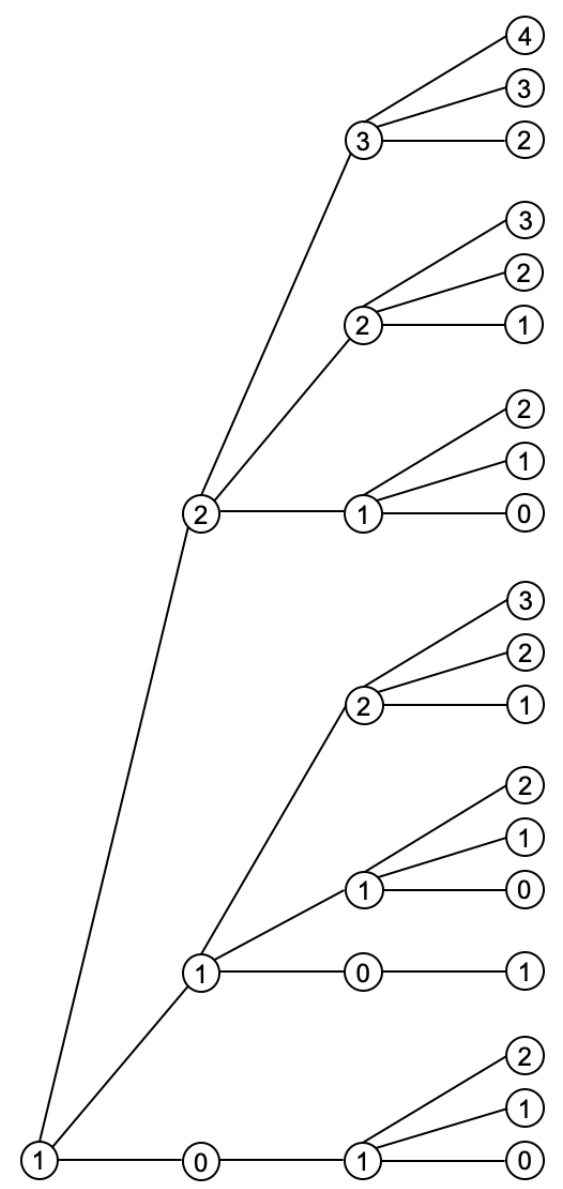

Figure 3: "Spin tree" representing the $S$ molecular spin states that are generated by the successive isotropic couplings of up to four $S=1$ single-site spin states. The summed number of $M_{S}$ components of these spin states define the size of the Heisenberg Hamiltonian $(81 \times 81)$.

size, ${ }^{42,63,64}$ in particular here zinc(II) ions to replace nickel(II) ones, (ii) use ab initio embedding model potentials ${ }^{65}$ to mimic the important electrostatic effects driven by the presence of the replaced paramagnetic ions ${ }^{42}$ (iii) consider the other paramagnetic ions in their ground closed-shell configuration, ${ }^{7}$ or (iv) lock other paramagnetic ions in a high spin state described with only one configuration. ${ }^{66}$ Independent of the retained approach, only one paramagnetic ion is considered "active" and one may then focus attention to the ZFS of the $S=1$ state of the ion of interest.

The description of the ZFS of an $S=1$ states can be made by applying a simple spin Hamiltonian: 62,67 
Table 1: The solutions of the equation 2, adapted from Reference 18. The states are characterized by an $S$ quantum number and by an index $n$ for each $S$ block.

\begin{tabular}{ccc}
\hline$S$ & $n$ & Heisenberg \\
\hline 4 & 1 & 0 \\
3 & 1 & $-4 J_{1}$ \\
3 & 2 & $-2 J_{1}-2 J_{2}$ \\
3 & 3 & $-2 J_{1}-2 J_{2}$ \\
2 & 1 & $-7 J_{1}$ \\
2 & 2 & $-5 J_{1}-2 J_{2}$ \\
2 & 3 & $-5 J_{1}-2 J_{2}$ \\
2 & 4 & $-4 J_{1}-3 J_{2}$ \\
2 & 5 & $-4 J_{1}-3 J_{2}$ \\
2 & 6 & $-3 J_{1}-4 J_{2}$ \\
1 & 1 & $-9 J_{1}$ \\
1 & 2 & $-7 J_{1}-2 J_{2}$ \\
1 & 3 & $-7 J_{1}-2 J_{2}$ \\
1 & 4 & $-5 J_{1}-4 J_{2}$ \\
1 & 5 & $-4 J_{1}-5 J_{2}$ \\
1 & 6 & $-4 J_{1}-5 J_{2}$ \\
0 & 1 & $-10 J_{1}$ \\
0 & 2 & $-6 J_{1}-4 J_{2}$ \\
0 & 3 & $-4 J_{1}-6 J_{2}$ \\
\hline
\end{tabular}

$$
\hat{\mathbf{H}}^{S=1}=\hat{\mathbf{S}} \overline{\bar{D}} \hat{\mathbf{S}}
$$

where $\hat{\mathbf{S}}$ is the spin operator vector and $\overline{\bar{D}}$ the ZFS tensor. Without surprise, this Hamiltonian was succesfully confronted to state-of-the-art effective Hamiltonians, ${ }^{30}$ meaning that this model phenomenologically built perfectly captures all the important information contained in the many-electron states and energies (see Section 2.6 for more details concerning this procedure). The $\overline{\bar{D}}$ tensor being symmetric, it can be diagonalized to obtain the magnetic anisotropy axis frame and its diagonal elements in this frame. In the magnetic anisotropy axis frame, $\hat{\mathbf{H}}^{S=1}$ can be rewritten as:

$$
\hat{\mathbf{H}}^{S=1}=D\left(\hat{S}_{z}^{2}-\frac{2}{3}\right)+E\left(\hat{S}_{x}^{2}-\hat{S}_{y}^{2}\right)
$$

where $D$ is the axial ZFS parameters, defined as: 


$$
D=D_{z z}-\frac{1}{2}\left(D_{x x}-D_{y y}\right)
$$

and $E$ the rhombic one, defined as:

$$
E=\frac{1}{2}\left(D_{x x}-D_{y y}\right)
$$

and where conventions apply to define the main anisotropy axis and label the two other ones, as for instance:

$$
0 \leq \frac{E}{D} \leq \frac{1}{3}
$$

In this convention, used in this work, $D$ and $E$ have the same sign. For the sake of completeness, one should mention that an alternative convention is also widely spread, for which $E$ is positive defined:

$$
\frac{E}{|D|} \leq \frac{1}{3}
$$

In both the cases, the spirit is the same, the main anisotropy axis is the most distinct one. In the complex under study, no local symmetry element constraints the orientations of the single-site magnetic anisotropy axes. Thus, these will be an actual output of the performed quantum mechanical calculations.

Within multiconfigurational and relativistic frameworks, the determination of the ZFS of $S=1$ states require to define a configuration interaction (CI) space consisting of the $M_{S}$ components of a set of non-relativistic or scalar-relativistic states. As mentioned earlier, the first coordination spheres of the nickel(II) ions of interest consist in distorted octahedra. Due to the $\mathrm{S}_{4}$ symmetry, the single-site anisotropy can be computed with any of the nickel(II) ion as active. For computing this single-site anisotropy, the CI space must be designed in such a way that it would respect the isotropy of the octahedral symmetry. In practice, this 
means taking the analoguous of entire multiplets in the octahedron. Therefore, several set of states may be employed. As previously shown, ${ }^{30}$ one may then consistently consider $4 S=$ 1 states, $7 S=1$ states and $2 S=0$ ones, or the entire $\mathrm{d}^{8}$ manifold (with 8 electrons within 5 orbitals, one may build $10 S=1$ states and $15 S=0$ ones). These three CI spaces will be considered in this work. Note that the rationalization of the ZFS in model situations can be safely done with $4 S=1$ states by means of second order perturbation theory. ${ }^{29,68}$ This space is not randomly chosen: it includes the lowest-lying single-excited states, meaning that the numerators can be quite large (the SOC operator is essentially a monoelectronic one, thus it includes the excited states that most couple with the ground one), and so do the denominators (simply because these are the lowest-lying states).

For applying equation 3, we suppose that the lowest-lying excited orbital states are well separated in energy with the ground state. In the case of a strict orbital degeneracy, even if the SOC between the components of the two orbitally degenerate states appears at second order of perturbations, ${ }^{69}$ the spin Hamiltonian approach is not suited anymore. Thus, a geometrical distortion is necessary to make the spin Hamiltonian approach relevant again. In the case of second-order SOC, even a tiny distortion can be enough, ${ }^{69}$ while the case of first-order SOC may require a more significant one. ${ }^{35}$

\subsection{The giant spin Hamiltonian for the ground $S=4$ state}

Providing that the ground $S=4$ state is separated enough in energy with the other spin states, the low-temperature magnetism can be described by a ZFS Hamiltonian acting only on the $M_{S}$ components of this state:

$$
\hat{\mathbf{H}}^{S=4}=\hat{\mathbf{S}} \overline{\bar{D}} \hat{\mathbf{S}}+\sum_{n=2}^{4} \sum_{q=-2 n}^{2 n} B_{2 n}^{q} \hat{O}_{2 n}^{q}
$$

where $\hat{\mathbf{S}}$ is the spin vector operator, $\overline{\bar{D}}$ the second-rank ZFS tensor (as before), $2 n$ the spin operator order, $q$ may be odd, and $B_{2 n}^{q}$ and $\hat{O}_{2 n}^{q}$ are the extended Stevens parameters and 
operators. ${ }^{68,70-72} 2 n$ cannot be larger than $2 S$, meaning that the summation in the complex of interest runs over the 4, 6 and 8 spin operator orders. In the $\mathrm{S}_{4}$ symmetry case, the main anisotropy axes is obvious: it is the $\mathrm{C}_{2} / \mathrm{S}_{4}$ symmetry axis. However, the tetragonal axis is

not known a priori. Assuming that the appropriate frame is determined, the $\hat{\mathbf{H}}^{S=4}$ model Hamiltonian may be rewritten as:

$$
\hat{\mathbf{H}}^{S=4}=D\left(\hat{S}_{z}^{2}-\frac{20}{3}\right)+\sum_{n=2}^{4}\left(B_{2 n}^{0} \hat{O}_{2 n}^{0}+B_{2 n}^{4} \hat{O}_{2 n}^{4}\right)
$$

where $D$ is the second-rank axial ZFS parameter (as before) and where negative $q$ indices are now prohibited (the problem is in fact recasted in terms of the standard Stevens operators). In practice, the summation may be stopped earlier as soon as a good representation of the spectrum is acquired. This approximation, typical in the interpretation of experimental data, will be also discussed here in terms of the outcomes of the quantum mechanical calculations (see Results Section). Indeed, the use of the effective Hamiltonian approach (see below) may allow one to use the information contained in the wave functions and energies and unambiguously discard or even extract ${ }^{43,69}$ the values of such parameters.

\subsection{Ab initio calculations: Methods and details}

The multiconfigurational and relativistic calculations performed in this work are based on a two-step approach in which a set of scalar-relativistic states is first computed prior to diagonalizing a spin-orbit configuration interaction (SOCI) matrix, expressed in the basis of the $M_{S}$ components of the scalar-relativistic states. The Douglas-Kroll-Hess (DKH) Hamiltonian is used to explicitly account for scalar relativistic effects. ${ }^{73-75}$ The scalar relativistic states are computed at the state-average complete active space self-consistent field (CASSCF) level. ${ }^{76,77}$ Four different active spaces will be considered:

- isotropic couplings on the full complex: minimal active space consisting of 8 electrons in 8 orbitals, i.e. the magnetic electrons and orbitals for four nickel(II) sites 
- isotropic couplings on clusters with two active magnetic sites: minimal active space consisting of 4 electrons in 4 orbitals, i.e. the magnetic electrons and orbitals for two nickel(II) sites

- single-ion anisotropies: minimal active space consisting of 8 electrons in 5 orbitals, i.e. the d orbitals and electrons for one nickel(II) site

- molecular anisotropy of the ground $S=4$ state: minimal active space consisting of 32 electrons in 20 orbitals, i.e. the d orbitals and electrons for four nickel(II) sites

Since state-average calculations will be performed, the results naturally depend on the number of states that are included in the calculations. In the case of the isotropic couplings, these will include the full Heisenberg space (see Figure 3 and Table 1) and in the case of the full complex also a subset of it $(1 S=4+3 S=3$ states $)$. In the case of the single-ion anisotropies, three spaces will be considered (see Section 2.3). Concerning the ZFS of the ground $S=4$ state, two space will be considered and detailed in the Results Section since these will be based on the previous results on the single-ion anisotropies.

Since the state-average CASSCF calculations may not be good enough for capturing electron correlation, especially since minimal active spaces are considered (it is a pragmatical choice owing to the computational cost), multireference perturbation theory calculations will also be performed, based on the $N$-electron valence space perturbation theory at second order (NEVPT2) method. ${ }^{78-80}$ Although this approach may not be fully quantitative for computing isotropic couplings, it is quite successful for computing crystal-field excited states, thus for computing ZFSs. Actually, enlarging the active space with ligand orbitals displaying sizeable tails on the metal site may actually lead to an effective reduction of the ZFS parameters of up to $20 \%{ }^{30}$ On the other side, including the spin-spin coupling term may lead to an increase of a couple of $\%$ of the same values. ${ }^{17}$ Overall, the computed ZFS values are expected to be only slightly overestimated. For the sake of this article, it is important to show that the isotropic couplings are large enough to separate the ground $S=4$ state from the others belonging to 
the Heisenberg space. As a consequence, the tendency of NEVPT2 for underestimating the isotropic couplings ${ }^{55,56}$ should not be seen here as a pitfall of the present study. Naturally, this will be further commented on in the Results Section.

In the second step of the calculation, SOCI interaction matrices are diagonalized. Two main types of calculations will be performed, using (i) the CASSCF states and energies or (ii) the CASSCF states and the NEVPT2 energies to compute this matrix. Note that such a shift of the diagonal of the SOCI matrix as been first proposed by Teichtel et al. ${ }^{81}$ and later revisited by Llusar et al. ${ }^{82}$ Many succesful applications of this approach to compute the ZFS of nickel(II) ions have been reported in the literature (see for instance References 17, 31, 32, 34,38 and 40).

All the calculations reported in this article have been performed with ORCA v. 4.2.1. ${ }^{83}$ A mean-field SOC operator has been used ${ }^{84}$ together with the transformed SOC operator that is compatible with the DKH Hamiltonian (in other words the adequate "picture change" keyword has been switched on). DKH recontracted basis sets by D. A. Pantazis of the def2 type $^{85}$ have been used: TZVP for $\mathrm{Ni}$ and Zn atoms, i.e. (17s11p7d1f)/[10s6p4d1f] and (17s11p7d1f)/[10s7p3d1f], respectively, SVP for O, N and C atoms, i.e. (7s4p1d)/[3s2p1d], and SV for H atoms, i.e. (4s)/[2s]. Note that basis sets of similar sizes have been previously used to compute the ZFS of a nickel(II) complex. ${ }^{40}$ The def2-TZVP/C auxiliary basis set ${ }^{86}$ has been used for all the atoms. Since numerical accuracy can be critical to compute small energy differences and thus consistently extract weak effective parameters or small differences between them, convergence criteria must be wisely set up. In this work, the energy criterion (etol keyword) has been fixed to $10^{-8}$ (a.u.) and the gradient one (gtol keyword) to $10^{-4}$.

\subsection{Extraction of the model parameters and relevance of the model Hamiltonians}

As above-mentioned, the scalar-relativistic quantum mechanical calculations performed in this work consider spin-adapted solutions. Thus, these calculations are directly performed 
in the coupled $\left|S, M_{S}\right\rangle$ basis. When minimal active space CASSCF and post-CASSCF calculations are performed to compute isotropic couplings, the quantum mechanical solutions directly correspond to the solutions of the HDVV Hamiltonian (it is strictly diagonal in this basis). Thus, one can easily extract the isotropic couplings from eigenenergies of the $a b$ initio Hamiltonian. In this work, after shifting all the energies to set the one of the $S=4$ state to zero, the following expressions are used to compute the isotropic couplings in the full complex:

$$
J_{1}=-\frac{E(3,1)}{4}
$$

where $E(3,1)$ is the energy of the first $S=3$ state (the first index refers to $S$ and the second to $n$, as in Table 1), and:

$$
J_{2}=-\frac{1}{2}\left[\frac{E(3,2)+E(3,3)}{2}-\frac{E(3,1)}{2}\right]
$$

in which the mean value of the second and third $S=3$ state is taken to correct from the slight degeneracy lift that could occur in the quantum mechanical calculations (the calculations are performed in the $\mathrm{C}_{1}$ symmetry point group). In the clusters with only two active nickel(II) sites, a similar approach is employed:

$$
J_{i}=-\frac{E(1)}{2}
$$

where $i$ depends on the retained cluster according to equation $2, E(1)$ is the energy of the $S$ $=1$ state, obtained after having set energy of the $S=2$ state to the zero of the energy. In this case, only one index refering to $S$ is required since we have to deal only with one $S=2$, one $S=1$ and one $S=0$ state (see Figure 3 ).

After having extracted the $J$ values, one can easily compute the model spectra according to the expressions given in Table 1 (full complex) or simply following this expression (clusters with two active sites): 


$$
E(0)=-3 J_{i}
$$

where $J_{i}$ naturally depends again on the retained cluster. Mathematically, these model spectra have no strict reason to perfectly match the $a b$ initio energies. Thus, to assess the quality of the model, it is wise to define the error done by the model spectra, one way or another. In this work, we follow the expression previously used by Bastardis et al.: ${ }^{52}$

$$
\operatorname{Error}(\%)=\frac{\sum_{k=1}^{N}\left|E_{k}^{A b \text { initio }}-E_{k}^{\text {Model }}\right|}{N \Delta E^{A b \text { initio }}} \times 100
$$

where the mean absolute error done for each $k$ energy is given relative to the $a b$ initio spectral width $\Delta E^{A b}$ initio , which is here nothing but the energy of the highest state (the ground state is set at the zero of the energy).

The $\overline{\bar{D}}$ tensors are extracted with the effective Hamiltonian theory, ${ }^{87}$ based on the des Cloizeaux formalism: ${ }^{88}$

$$
\hat{\mathbf{H}}^{\text {des Cloizeaux }}=\sum_{k=1}^{N}\left|\widetilde{\Psi}_{k}^{\mathrm{L}}\right\rangle E_{k}\left\langle\widetilde{\Psi}_{k}^{\mathrm{L}}\right|
$$

where $E_{k}$ are the $a b$ initio energies and $\widetilde{\Psi}_{k}^{\mathrm{L}}$ are the Löwdin orthonormalized ${ }^{89}$ projections (onto the model space) of the $a b$ initio wave functions. Note that this approach has been first proposed by Maurice et al. ${ }^{30}$ to compute magnetic anisotropy parameters and is now implemented in $\mathrm{ORCA}^{83}$ for computing the second-rank ZFS parameters of $S$ states (no implementation is currently proposed for extracting the Stevens higher order parameters nor to extract parameters of multispin Hamiltonians). Diagonalization of the $\overline{\bar{D}}$ tensors will give the magnetic anisotropy axes and the $D$ and, if applicable, the $E$ parameters (see Section 2.3). Because only second-rank parameters are determined, the model spectra may not exactly match the $a b$ initio energies in the calculations dedicated to the ZFS of the $S=4$ state (full complex). Thus, the errors made by the model spectra will be computed according 
to equation 15. Furthermore, the expression of the effective Hamiltonians, given by ORCA in the arbitrary axis frames used for the calculations, will provide additional arguments for the importance of the neglected terms (in particular by looking at the off-diagonal elements of the effective Hamiltonians for the "tetragonal" parameters, e.g. $\left.B_{4}^{4}\right)$. Note that the application of the effective Hamiltonian approach to cases with magnetic sites displaying near orbital degeneracy may later lead to the definition of new model Hamiltonians, but that this task is clearly out of the scope of the present paper.

\section{Results}

\subsection{The isotropic couplings for the ground orbital configuration}

We start the discussion of the results with the computation of the isotropic couplings on the full complex, with four active nickel(II) sites, at the CASSCF(8/8) and NEVPT2 levels (see Tables 2 and 3). Though the calculations with $1 S=4$ and $3 S=3$ states already give access to the extraction of the $J_{1}$ and $J_{2}$ values, these cannot intrinsically lead to a serious discussion on the relevance of the HDVV Hamiltonian to describe the spectra corresponding to the full Heisenberg spaces. Thus, it is worth starting by looking at the results on the full Heisenberg spaces.

At the CASSCF(8/8) level, the model spectra gives an almost perfect description of the whole set of ab initio energies, as expected with state-average orbitals built with the full set of states $^{50}$ (in contrast with what could occur with state-specific orbitals or stateaverage orbitals per spin multiplicities). However, significant deviations are observed at the NEVPT2 level. These may have several origins, (i) they may be an artifact of multireference perturbation theory, ${ }^{55,56}$ (ii) they may reveal some biquadratic exchange couplings, ${ }^{51,53,62}$ or (iii) they may reveal even more complex effective interactions with for instance three-body

terms, which dominate the deviations in trinickel(II) systems. ${ }^{52}$ Due to the computational cost (the complex displays 104 atoms and the present basis set consists in 948 contracted 
Table 2: CASSCF (8/8) energies ("Ab initio", in $\mathbf{c m}^{-1}$ ), extracted $J_{1}$ and $J_{2}$ values $\left(\mathrm{cm}^{-1}\right)$, and model spectra ("Heisenberg", energies in $\mathrm{cm}^{-1}$ ). The states are characterized by an $S$ quantum number and by an index $n$ for each $S$ block. The error is given in \%.

\begin{tabular}{|c|c|c|c|c|c|c|c|c|c|}
\hline \multirow{2}{*}{$S$} & \multirow{2}{*}{$n$} & \multicolumn{4}{|c|}{$1 S=4+3 S=3$} & \multicolumn{4}{|c|}{ Full Heisenberg space } \\
\hline & & $A b$ initio & $J_{1}$ & $J_{2}$ & Heisenberg & $A b$ initio & $J_{1}$ & $J_{2}$ & Heisenberg \\
\hline 4 & 1 & 0.00 & -5.72 & -5.84 & 0.00 & 0.00 & -5.60 & -5.73 & 0.00 \\
\hline 3 & 1 & 22.87 & & & 22.87 & 22.42 & & & 22.42 \\
\hline 3 & 2 & 23.11 & & & 23.11 & 22.66 & & & 22.67 \\
\hline 3 & 3 & 23.11 & & & 23.11 & 22.67 & & & 22.67 \\
\hline 2 & 1 & & & & & 39.23 & & & 39.23 \\
\hline 2 & 2 & & & & & 39.48 & & & 39.48 \\
\hline 2 & 3 & & & & & 39.48 & & & 39.48 \\
\hline 2 & 4 & & & & & 39.60 & & & 39.60 \\
\hline 2 & 5 & & & & & 39.60 & & & 39.60 \\
\hline 2 & 6 & & & & & 39.72 & & & 39.73 \\
\hline 1 & 1 & & & & & 50.44 & & & 50.44 \\
\hline 1 & 2 & & & & & 50.68 & & & 50.69 \\
\hline 1 & 3 & & & & & 50.68 & & & 50.69 \\
\hline 1 & 4 & & & & & 50.93 & & & 50.93 \\
\hline 1 & 5 & & & & & 51.06 & & & 51.06 \\
\hline 1 & 6 & & & & & 51.06 & & & 51.06 \\
\hline 0 & 1 & & & & & 56.05 & & & 56.05 \\
\hline 0 & 2 & & & & & 56.54 & & & 56.54 \\
\hline \multirow[t]{2}{*}{0} & 3 & & & & & 56.78 & & & 56.79 \\
\hline & & & & Error & 0.00 & & & Error & 0.00 \\
\hline
\end{tabular}


Table 3: NEVPT2 energies ("Ab initio", in $\mathbf{c m}^{-1}$ ), extracted $J_{1}$ and $J_{2}$ values $\left(\mathrm{cm}^{-1}\right)$, and model spectra ("Heisenberg", energies in $\mathrm{cm}^{-1}$ ). The states are characterized by an $S$ quantum number and by an index $n$ for each $S$ block. The error is given in \%.

\begin{tabular}{|c|c|c|c|c|c|c|c|c|c|}
\hline \multirow{2}{*}{$S$} & \multirow{2}{*}{$n$} & \multicolumn{4}{|c|}{$1 S=4+3 S=3$} & \multicolumn{4}{|c|}{ Full Heisenberg space } \\
\hline & & $A b$ initio & $J_{1}$ & $J_{2}$ & Heisenberg & $A b$ initio & $J_{1}$ & $J_{2}$ & Heisenberg \\
\hline 4 & 1 & 0.00 & -5.63 & -5.92 & 0.00 & 0.00 & -5.59 & -5.88 & 0.00 \\
\hline 3 & 1 & 22.53 & & & 22.53 & 22.36 & & & 22.36 \\
\hline 3 & 2 & 23.11 & & & 23.10 & 22.94 & & & 22.94 \\
\hline 3 & 3 & 23.08 & & & 23.10 & 22.93 & & & 22.94 \\
\hline 2 & 1 & & & & & 38.67 & & & 39.14 \\
\hline 2 & 2 & & & & & 39.55 & & & 39.71 \\
\hline 2 & 3 & & & & & 39.55 & & & 39.71 \\
\hline 2 & 4 & & & & & 39.80 & & & 40.00 \\
\hline 2 & 5 & & & & & 39.79 & & & 40.00 \\
\hline 2 & 6 & & & & & 39.58 & & & 40.28 \\
\hline 1 & 1 & & & & & 49.21 & & & 50.32 \\
\hline 1 & 2 & & & & & 50.10 & & & 50.89 \\
\hline 1 & 3 & & & & & 50.09 & & & 50.89 \\
\hline 1 & 4 & & & & & 50.56 & & & 51.46 \\
\hline 1 & 5 & & & & & 50.11 & & & 51.75 \\
\hline 1 & 6 & & & & & 50.08 & & & 51.75 \\
\hline 0 & 1 & & & & & 54.06 & & & 55.91 \\
\hline 0 & 2 & & & & & 55.50 & & & 57.06 \\
\hline \multirow[t]{2}{*}{0} & 3 & & & & & 54.03 & & & 57.63 \\
\hline & & & & Error & 0.02 & & & Error & 1.44 \\
\hline
\end{tabular}


basis functions and the size of the active space leads to a huge amount of configurations for the lower spin cases), it is practically impossible to perform multireference CI calculations to distinguish the true physics from artifacts of multireference perturbation theory.

To somehow quantify the deviations, calculations have been performed in clusters with only two active nickel(II) sites. In cluster $\mathbf{1}, \mathrm{Ni}_{1}$ and $\mathrm{Ni}_{2}$ are active and $\mathrm{Ni}_{3}$ and $\mathrm{Ni}_{4}$ are replaced by zinc(II) ions, while in cluster $2 \mathrm{Ni}_{1}$ and $\mathrm{Ni}_{3}$ are active and $\mathrm{Ni}_{2}$ and $\mathrm{Ni}_{4}$ are replaced by zinc(II) ions. This notation is chosen to match the indices of equation 2. Similarly, no sizeable deviation to the Landé intervals is observed at the CASSCF(4/4) levels, contrary to what happens at the NEVPT2 ones.

Table 4: CASSCF energies ("Ab initio", in $\mathbf{c m}^{-1}$ ), extracted $J_{1}$ and $J_{2}$ values $\left(\mathbf{c m}^{-1}\right)$ and $\lambda_{1}$ and $\lambda_{2}$ parameters, and model spectra (Heisenberg", energies in $\left.\mathrm{cm}^{-1}\right)$. The states are characterized by an $S$ quantum number. The error is given in $\%$.

\begin{tabular}{ccccccccc}
\hline \multirow{2}{*}{$S$} & \multicolumn{3}{c}{ Cluster 1 } & & \multicolumn{4}{c}{ Cluster 2 } \\
& Ab initio & $J_{1}$ & Heisenberg & $\lambda_{1}$ & Ab initio & $J_{2}$ & Heisenberg & $\lambda_{2}$ \\
\hline 2 & 0.00 & -5.50 & 0.00 & 0.00 & 0.00 & -5.63 & 0.00 & 0.00 \\
1 & 11.00 & & 11.00 & & 11.26 & & 11.26 & \\
0 & 16.50 & & 16.50 & & 16.88 & & 16.88 & \\
& & Error & 0.00 & & & Error & 0.00 & \\
\hline
\end{tabular}

Table 5: NEVPT2 energies ("Ab initio", in $\mathbf{c m}^{-1}$ ), extracted $J_{1}$ and $J_{2}$ values $\left(\mathrm{cm}^{-1}\right)$ and $\lambda_{1}$ and $\lambda_{2}$ parameters, and model spectra ("Heisenberg", energies in $\left.\mathrm{cm}^{-1}\right)$. The states are characterized by an $S$ quantum number. The error is given in $\%$.

\begin{tabular}{ccccccccc}
\hline \multirow{2}{*}{$S$} & \multicolumn{3}{c}{ Cluster 1 } & \multicolumn{5}{c}{ Cluster 2 } \\
& Ab initio & $J_{1}$ & Heisenberg & $\lambda_{1}$ & Ab initio & $J_{2}$ & Heisenberg & $\lambda_{2}$ \\
\hline 2 & 0.00 & -5.40 & 0.00 & 0.02 & 0.00 & -5.58 & 0.00 & 0.04 \\
1 & 10.80 & & 10.80 & & 11.15 & & 11.15 & \\
0 & 15.90 & & 16.20 & & 16.03 & & 16.73 & \\
& & Error & 0.64 & & & Error & 1.44 & \\
\hline
\end{tabular}

Following the same reasoning as Moreira et al., ${ }^{51}$ it is proposed to quantify the observed deviations to the HDVV Hamiltonian by introducing an effective biquadratic exchange term as follows: 


$$
\hat{\mathbf{H}}^{\mathrm{HDVV}}=J_{i j}\left(\hat{\mathbf{S}}_{i} \cdot \hat{\mathbf{S}}_{j}+\lambda_{i j}\left(\hat{\mathbf{S}}_{i} \cdot \hat{\mathbf{S}}_{j}\right)^{2}\right)
$$

where $\lambda_{i j}$ corresponds to the relative deviation to the HDVV Hamiltonian, and where $\mathrm{J}_{i j}$ is the coupling constant derived from the energies of the $S=2$ and $S=1$ states. Note that to be consistent with the previous notations, Tables 4 and 5 refer to the $\lambda_{1}$ and $\lambda_{2}$ parameters. As soon as two parameters are now introduced for reproducing two energy differences, no error can now be atributted to the model Hamiltonian, hence no reported values for this model Hamiltonian in these tables. The derived parameters are all smaller than to 0.05, a value that is a limit for the acceptable physical deviations. ${ }^{62}$ However, we recall here that we do not know at this stage if these deviations are physical and/or artifactual, the extracted parameters should thus be only interpreted as effective deviations in the computed spectra, and not in terms of "true" biquadratic exchange parameters.

A more important result for the sake of this article dedicated to the ZFS of the ground $S=4$ state is the semi-quantitative estimates for the isotropic couplings. At all the considered levels of theory, $J_{1}$ and $J_{2}$ values of about $-5.7 \pm 0.4 \mathrm{~cm}^{-1}$ are obtained (the mean value of all the computed isotropic couplings has been taken together with two standard of deviations). Consequently, even if we underestimate the isotropic couplings at the considered CASSCF and NEVPT2 levels, the ground $S=4$ state is always separated to the first $S=3$ state by more than $20 \mathrm{~cm}^{-1}$ in the computed spectra. This energy separation, mentioned elsewhere in the analysis of magnetic data, ${ }^{18}$ is of crucial importance to limit the spin mixings and the energy overlap between the energy levels of states displaying different spin multiplicities. In other words, it is a good start for justifying the applicability of the giant spin approximation for describing the low-temperature magnetism of the complex. Furthermore, the computed values confirm weak ferromagnetic couplings in this system, in agreement with experiment ${ }^{19}$ and with the previous DFT results. ${ }^{22}$

No matter the system (full complex vs. cluster) or the method (CASSCF or NEVPT2), the computed values for $J_{1}$ and $J_{2}$ are always close one another. This result corroborates the 
DFT results of Venegas-Yazigi et al., ${ }^{22}$ who have already pointed out the discrepancy between the experimental structure and the experimental fit of the magnetic data (see Discussion Section).

Finally, it is important to note that the results obtained with $1 S=4$ and $3 S=3$ states fairly match the ones obtained with the full Heisenberg space in Tables 2 and 3, meaning that the $J_{1}$ and $J_{2}$ values for the ground orbital configuration can be safely extracted on the basis of these states.

\subsection{The single-site anisotropies}

The single-site anisotropies being symmetry equivalent in the full complex $\left(\mathrm{S}_{4}\right.$ symmetry), a cluster containing only one active nickel(II) site is enough to give all the necessary information. In practice, $\mathrm{Ni}_{1}$ was considered active while $\mathrm{Ni}_{2}, \mathrm{Ni}_{3}$ and $\mathrm{Ni}_{4}$ have been replaced by zinc(II) ions. Three state-averaging spaces have been considered, $4 S=1,7 S=1+2 S$ $=0$ and the full $8 / 5$ space consisting of $10 S=1$ and $15 S=0$ states (see Table 6 ). In all the reported results, $E / D$ is close to the $1 / 3$ limit, meaning that the $D$ sign is not actually determined by the $a b$ initio calculations, which is an unfortunate situation that has already been encountered in a mononuclear nickel(II) complex. ${ }^{32}$

Table 6: The SOCI single-site anisotropy parameters. $D$ is given in $\mathbf{c m}^{-1}, E / D$ is positive defined, $\vartheta\left({ }^{\circ}\right)$ is the angle between the single-site easy axis of magnetization and the $\mathrm{Ni}_{1}-\mathrm{Ni}_{3}$ (or $\mathrm{Ni}_{2}-\mathrm{Ni}_{4}$ ) orientation and $\delta\left(^{\circ}\right.$ ) is the torsion angle between the $\mathrm{Ni}_{1}$ and $\mathrm{Ni}_{3}$ (or $\mathrm{Ni}_{2}$ and $\mathrm{Ni}_{4}$ ) single-site easy axes of magnetization.

\begin{tabular}{ccccccccccccc}
\hline \multirow{2}{*}{ Energies } & \multicolumn{4}{c}{$4 S=1$} & \multicolumn{4}{c}{$7 S=1+2 \mathrm{~S}=0$} & \multicolumn{4}{c}{ Full 8/5 space } \\
& $D$ & $E / D$ & $\vartheta$ & $\delta$ & $D$ & $E / D$ & $\vartheta$ & $\delta$ & $D$ & $E / D$ & $\vartheta$ & $\delta$ \\
\hline CASSCF & 2.78 & 0.32 & 65.1 & 10.1 & -2.88 & 0.31 & 63.8 & 11.0 & -2.67 & 0.31 & 64.5 & 10.1 \\
NEVPT2 & 2.38 & 0.33 & 65.2 & 10.1 & -2.40 & 0.30 & 63.6 & 9.4 & -2.03 & 0.31 & 63.3 & 9.3 \\
\hline
\end{tabular}

Since the $D$ parameter for the $S=4$ state of the full complex is known to be negative from experiment, ${ }^{19}$ it is interesting to monitor the position of the single-site easy axis of magnetization. Its orientation is here given in terms of two angles, $\vartheta$ being the angle between the $\mathrm{Ni}_{1}-\mathrm{Ni}_{3}$ orientation and this axis and $\delta$ being the torsion angle between the $\mathrm{Ni}_{1}$ easy 
axis of magnetization and the one of $\mathrm{Ni}_{3}$ obtained by applying the $\mathrm{C}_{2}$ symmetry operation to the $\mathrm{Ni}_{1}$ easy axis. As can be seen in Table 6, the orientation of the single-site easy axis of magnetization is similar at all the considered levels of theory, meaning that it can be discussed at any of these levels.

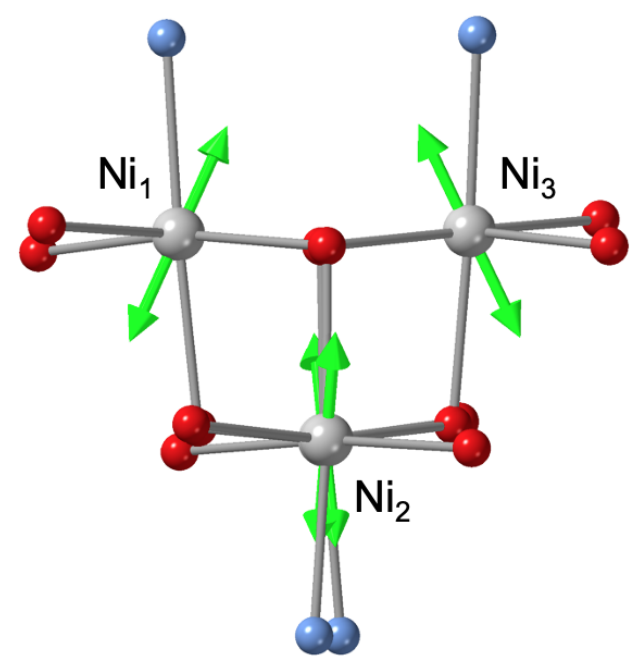

Figure 4: Representation of the single-site easy axes of magnetization (double sided arrows). The $\mathrm{Ni}_{1}$ axis was obtained at the SOCI level with the $M_{S}$ components of the CASSCF $(8 / 5)$ states $(4 S=1)$ and the others by applying the adequate symmetry operations $\left(\mathrm{Ni}_{2}-\mathrm{Ni}_{4}\right)$. The $\mathrm{C}_{2}$ symmetry axis is vertical in the paper plane. Color code: gray stands for $\mathrm{Ni}$, red for $\mathrm{O}$ and blue for $\mathrm{N}$.

In Figure 4, the single-site easy axis of magnetization obtained at the CASSCF $(8 / 5)$ level with $4 S=1$ states are depicted. These easy axes are far from leading to a favorable situation to maximize the anisotropy of the full complex, as would be the case for instance if these would be parallel. ${ }^{7}$ This is already a first strong explanation for the weak uniaxial anisotropy of the $S=4$ state.

In appearance, the computed parameters are significantly dependent on the number of states that is included in the calculation. Since the scalar-relativistic states of the first step of the calculation are all computed with a state-averaging scheme, inclusion of more states is expected on the one side to account for more physical contributions in the second step of the calculation and on the other side to enlarge the state-averaging artifacts, as already pointed 
out by Maurice et al. in 2009. ${ }^{30}$ To sort out both contributions, the individual contributions of the excited states have been computed with second order perturbation theory with the 7 $S=1$ and $2 S=0$ space (see Table 7 ). As can be seen, the lowest three $S=1$ states, already present in the $4 S=1$ space in Table 6 , largely dominate the computed ZFS parameters (the contributions of the next three $S=1$ states and of the lowest two $S=0$ states being only marginal). Thus, it is concluded that the $4 S=1$ space is the best one for computing the single-site ZFS, since it is the one that account for the actual physical contributions to it, while keeping to a minimum the averaging artifacts (the inclusion of more states necessarily comes with more averaging artifacts). Note that this is due to the fact that the first coordination spheres of the nickel(II) ions in the complex under study are somehow close to the octahedron case, i.e. this conclusion may not be transferable to every $\mathrm{Ni}_{4}$ complexes. However, in this case, it is actually an important result to open the way for the calculation of the ZFS of the ground $S=4$ state.

Table 7: Individual contributions $\left(\right.$ in $\left.\mathrm{cm}^{-1}\right)$ at the second order of perturbation theory to the single-site anisotropy parameters.

\begin{tabular}{cccccc}
\hline \multicolumn{2}{c}{ Energies } & \multicolumn{2}{c}{ CASSCF } & \multicolumn{2}{c}{ NEVPT2 } \\
$S$ & $n$ & $D$ & $E$ & $D$ & $E$ \\
\hline 1 & 2 & -28.00 & 3.15 & -21.78 & 1.44 \\
1 & 3 & 2.18 & -5.48 & 1.26 & -5.68 \\
1 & 4 & 22.68 & 1.38 & 17.97 & 3.48 \\
1 & 5 & -0.02 & 0.00 & -0.01 & 0.00 \\
1 & 6 & -0.02 & 0.00 & -0.02 & 0.00 \\
1 & 7 & 0.01 & 0.00 & 0.01 & 0.00 \\
0 & 1 & 0.00 & 0.00 & 0.00 & 0.00 \\
0 & 2 & 0.00 & 0.00 & 0.00 & 0.00 \\
\multicolumn{2}{c}{ Total } & -3.17 & -0.94 & -2.57 & -0.76 \\
\hline
\end{tabular}

\subsection{The zero-field splitting of the ground $S=4$ state}

As mentioned before, the single-site anisotropies are better computed with the $4 S=1$ states. In the full complex, this would mean in principle considering twelve single excitations from the ground $S=4$ state, generating $13 S=4$ states, and many other states of lower spin 
multiplicities with one complete Heisenberg spectrum per single excitation. However, the SOC operator can only couple at the second order of perturbations states with same spins or spins differing only by one from the reference spin multiplicity. Here, this means that we can focus more specifically on the $S=3$ manifold, and neglect the $S=2, S=1$ and $S=0$ ones. According to Figure 3, $3 S=3$ states are associated with $1 S=4$ one. Consequently, a good space for computing the ZFS of the ground $S=4$ state would consist in $13 S=4$ states and $39 S=3$ ones. Test calculations with larger numbers of $S=4$ and/or $S=3$ states have revealed that the $13 S=4$ and $39 S=3$ states are well separated in energy from the higher ones, meaning that these states have their anticipated meaning (single-excited states with respect to the ground $S=4$ state). Another argument confirming this may come from the average occupation numbers of the state average orbitals. Numbers very close to the ideal $25 / 13=1.92$ and $29 / 26=1.12$ values that can be computed assuming pure single excitations are actually obtained at the CASSCF level (not shown). Note that with only one active site, those ideal numbers would be $7 / 4=1.75$ and $11 / 8=1.37$, respectively, and that numbers close to these were actually obtained in Section 3.2 (with $4 S=1$ states, naturally).

Table 8: The molecular anisotropy parameters for the ground $S=4$ state computed at the SOCI level with the $M_{S}$ components of the CASSCF $(32 / 20)$ states. $D$ is given in $\mathbf{c m}^{-1}, E / D$ is positive defined and the error is given in \%.

\begin{tabular}{ccccccc}
\hline \multirow{2}{*}{ Energies } & \multicolumn{3}{c}{$13 S=4$} & \multicolumn{3}{c}{$13 S=4+39 S=3$} \\
& $D$ & $E / D$ & Error & $D$ & $E / D$ & Error \\
\hline CASSCF & -0.41 & 0.00 & 0.04 & -0.22 & 0.00 & 0.26 \\
NEVPT2 & -0.33 & 0.00 & 0.03 & $\mathrm{NC}^{1}$ & $\mathrm{NC}$ & $\mathrm{NC}$ \\
\hline
\end{tabular}

${ }^{1} \mathrm{NC}$ stands for not computed, here owing to the prohibitive computational cost.

In Table 8, two spaces are considered, $13 S=4$ states (same spin contributions) and 13 $S=4+39 S=3$ states (same spin plus contributions from the $S-1$ spin states). Before commenting on the $D$ values, it is worth checking if the neglect of the higher order Stevens terms is an issue or not. For this, the model spectra obtained by neglecting these terms have been confronted to the $a b$ initio energies, as was done for the isotropic couplings (see Section 3.1). As can be seen in Table 8, the committed errors are actually negligible when 
only $S=4$ states are included, and it remains quite moderate after inclusion of $S=3$ states. Furthermore, the des Cloizeaux effective Hamiltonians ${ }^{88}$ give no sign of tetragonal terms such as $B_{4}^{4}$, which would effectively couple $M_{S}$ and $M_{S} \pm 4$ energy levels, and potentially break the degeneracy of $\pm M_{S}$ levels. As a conclusion, neglecting the higher order Stevens terms is not an issue here and the following model Hamiltonian appears fairly suited to reproduce the $a b$ initio spectra:

$$
\hat{\mathbf{H}}^{S=4}=D\left(\hat{S}_{z}^{2}-16\right)
$$

where the zero of the energy has been set to the ground $M_{S}= \pm 4$ energy levels.

The results with the $13 S=4$ states show a significant role of the additional electron correlation delivered by NEVPT2 on top of CASSCF. Unfortunately, performing NEVPT2 calculations with $13 S=4+39 S=3$ states was not an option due to the associated computational cost. However, one may expect also some significant contribution. Note that the inclusion of the $39 S=3$ states leads to an important reduction of the $D$ parameter, and that this is a true physical effect and not an averaging artifacts, test calculations having shown at second order of perturbation theory that these states do contribute to the ZFS. Since the contributions of the two $S=4$ and $S=3$ spin blocks overall oppose, the reduction observed with NEVPT2 with the $13 S=4$ states cannot be quantitatively transfered to estimate the one that would have been obtained with $13 S=4+39 S=3$ states. However, one can expect that the resulting value would significantly deviate from the experimental value of $-0.43 \mathrm{~cm}^{-1}$, most probably being lower than it.

It is worth mentioning that a $\operatorname{CASSCF}(32 / 20)$ calculation performed with $25 S=4$ states has lead to a $D$ value of $-0.41 \mathrm{~cm}^{-1}$ (as with $13 S=4$ states), and that second order perturbation theory has shown that the added individual contributions to the $D$ parameter are negligible, following what was obtained with the next three $S=1$ states for the single-ion anisotropy parameters. Thus, the $13 S=4+39 S=3$ space is indeed fully consistent for computing this ZFS. 
Finally, the $13 S=4$ and $39 S=3$ space also gives access to the $J_{1}$ and $\mathrm{J}_{2}$ values for the ground orbital configuration. From the energies of the lowest $S=4$ and the lowest three $S=3$ states, at the CASSCF (32/20) level, $J_{1}$ and $J_{2}$ values of -5.80 and $-5.86 \mathrm{~cm}^{-1}$, respectively, are obtained, in satisfactory agreement with the previous CASSCF estimates (see Tables 2 and 4) and also in practice with the NEVPT2 ones (see Tables 3 and 5).

\section{Discussion}

In the experimental study of Moragues-Canovas et al. ${ }^{19}$ two quite distinct isotropic couplings were obtained, $J_{1}=-8 \mathrm{~cm}^{-1}$ and $J_{2}=-15 \mathrm{~cm}^{-1}$ (note the change in sign for consistency with equation 2). Moreover, two parameter sets were found to satisfactorily fit the magnetic data: $D=-0.43 \mathrm{~cm}^{-1}$ and $E / D=0.04$ and $D=-0.48 \mathrm{~cm}^{-1}$ and $E / D=0.23$. The authors concluded that the second set was not probable, on the basis that the structure is not meant to display a strong rhombicity (it is strictly zero in the $\mathrm{S}_{4}$ symmetry point group). Thus, the new results, together with the previous DFT study concerning solely the isotropic couplings, ${ }^{22}$ support a discrepancy between the reported structure and the magnetic parameters.

Such a discrepancy is not completely new in the field of molecular magnetism, and may arise for instance from a temperature difference (the crystal structure may not be determined at the same temperature as the one used for deriving the magnetic parameters) or simply by averaging effects as suggested for instance in a joint theory/experiment study concerning the nickel(II) mononuclear complex for which the crystal structure has been determined

at a temperature as low as $10 \mathrm{~K} .{ }^{35}$ It is thus always difficult to experimentally determine local distortions that actually lower the molecular symmetry. Note that other examples of structures questioned by quantum mechanical calculations are available, see for instance Reference 20 .

Though it is obvious that only a symmetry lowering can explain the occurrence of a 
rhombic parameter in the complex under study, it is hard to know a priori if such an effect may actually enlarge $D$ (the best estimate in this work is lower than the experimental value by about a factor of 2 , which is actually similar to what was encountered in a previous work on a dinickel(II) complex $\left.{ }^{29,43,44}\right)$. To illustrate how much the $D$ parameter can be sensitive to the structure, preliminary $\operatorname{CASSCF}(32 / 20)$ calculations have been performed on an alternative structure (not reported) only differing from the one used in this work by the positions of the eight missing $\mathrm{H}$ atoms. The positions of these $\mathrm{H}$ atoms has also been optimized with Crystalmaker, ${ }^{45}$ but starting with random positions that do not fulfil the $\mathrm{S}_{4}$ symmetry. The resulting $D$ parameter, obtained with the $13 S=4$ space, -0.51 $\mathrm{cm}^{-1}$, is significanly larger in absolute value than the one obtained in Table 8 with the same space, while only the positions of the tripodal ligand terminations are changed. Also, the obtained $E / D$ parameter, 0.05 , is more in line with the experimental values. Therefore, it is concluded that the main source of discrepancy between the results reported in Table 8 and the expeirmental ones ${ }^{19}$ must come from either the structure itself or from the use of a single structure. Without performing a complete new study on this, it is practically impossible to say if the problem can be solved with one single optimized structure (presumably not solely resulting from a simple gas phase optimization of the isolated complex due to the resulting neglect of environment effects) or by considering an ensemble of discrete structures coming for instance from snapshots of molecular dynamics simulations, as done for instance to compute ionization energies of solvated halides by Bouchafra et al. ${ }^{90}$

A major issue in the molecular magnetism community relates to the link between the single-site anisotropies and the molecular ones. As shown in a previous paper, ${ }^{7}$ it is very tedious to do so even with only two nickel(II) sites. Also, deriving the contributions of the single-site anisotropies to a molecular one (while neglecting the intersite anisotropies) is quickly cumbursome, unless the single-site anisotropy axes coincide (by being collinear or parallel) or are coplanar with a tilting angle ${ }^{29,91}$ or orthogonal. ${ }^{7,92}$ Figure 4 clearly shows that the complex under study to not correspond to such a specific case (with ideal angles 
for instance) but rather to the general one. Without pretending that it is impossible to attempt something, I believe that such a technical task with four single-site tensors would go far beyond the scope of this article that aim at showing what is practically and readily computable in the case of a real and polynuclear single-molecule magnet. Moreover, since it has been shown that the standard multispin Hamiltonian is questionable already in binuclear systems ${ }^{42}$ and that no simple relation appears in the general case between the single-site anisotropy parameters and the molecular ones, ${ }^{7}$ the interest for such a quantitative task is also questionable. Instead, I believe that it is more useful to directly compute the giant-spin anisotropy parameters, as done here in the case of a tetranickel(II) complex, and to keep the discussion on the relationship between the single-site anisotropies and the molecular one at a qualitative level by for instance commenting on the relative orientations of the single-site anisotropy axes, which is fairly doable whatever the cluster topology is.

The natures of the lowest-lying excited states have not been given here for the sake of simplicity, even out of the calculations with only one active nickel(II) site. As shown in Figure 4, the single-site anisotropy axes do not match at all the coordination bonds between the active nickel(II) site and its first-coordination sphere atoms. As a consequence, the nature of the states in this frame cannot match the simple ones that occurs in ideal $D_{2 \mathrm{~h}}$ situations. ${ }^{29}$ Actually, in the magnetic axis frame, while the ground $S=1$ state is clearly single-configurational, the expressions of the two magnetic orbitals for this state already involve all the valence $\mathrm{d}$ functions $\left(\mathrm{z}^{2}, \mathrm{xz}, \mathrm{yz}, \mathrm{x}^{2}-\mathrm{y}^{2}\right.$ and $\left.\mathrm{xy}\right)$ and the wave functions of the three lowest-lying excited triplets all appear multiconfigurational with CI coefficient values that are not ideal. When one enters in such situations that are general and that do not simplify by symmetry, the pen and paper approach strongly loses its interest, if not becoming impossible. Again, this is a typical case for showcasing the interest for performing quantum mechanical calculations. Even if their outcomes may not be fully elucidated, as it is the case here, they provide useful clues to the investigators by allowing them to determine the single-ion anisotropy parameters and axes. 


\section{Perspectives}

Several methodological perspectives of interest for the molecular magnetism community arise from this work. First, it would be interesting to know if actual deviations to the HDVV Hamiltonian can occur in $\mathrm{Ni}_{4}$ complexes, and if so, if four-body terms ${ }^{51}$ are at play. For this, adequate multirefence CI calculations would have to be performed, in the same vein of what was done for $\mathrm{Ni}_{3}$ complexes to reveal three-body terms. ${ }^{52}$ In the same vein, the effective Hamiltonian approach will have to be applied to more cases with near orbital degeneracy to question and maybe propose alternatives to the spin Hamiltonian formalism.

Second, an extensive structural analysis could be performed to rationalize potential discrepancies between the experimental data and the computed ones. Actually, these may appear in any type of molecular complex, and not only in $\mathrm{Ni}_{4}$ ones as the one studied in this work. In particular, it should be noted that although good agreement can be obtained for the axial $D$ parameter, the rhombic $E$ one is more enclined to be a source of trouble already for mononuclear complexes nickel(II) complexes, ${ }^{30-32,40}$ while the case of a nickel(II) complex is meant to be the simplest situation for the quantum mechanical calculations. This may pave the way toward structural studies that go beyond the common habit of using only one structure, and that, if an experimental one exists, to use that one without further conditions (though partial or complete geometry optimizations with DFT have already been seen in the literature, as for instance in Reference 93).

Third, it is worth mentioning that this work constitutes a first step toward the theoretical determination of anisotropic Hamiltonians in polynuclear complexes with higher single-site spins and/or more magnetic sites, and with any cluster topology. Indeed, the proposed methodology, based on multiconfigurational calculations and the des Cloizeaux effective Hamiltonian formalism ${ }^{88}$ is generally applicable independent on the single-site spin quantum numbers and the number of magnetic sites. However, two main issues may appear in practice, the necessity for keeping the number of states to a sizeable amount for avoiding large averaging artifacts (and calculations with prohibitive computational costs), and the 
necessity for handling large active spaces. Perhaps the use of the density matrix renormal-

ization group technique, as used for instance by Roemelt et al., ${ }^{57}$ will later prove to be a route toward this.

Finally, the fact that we are now capable of directly treating the molecular anisotropy of a tetranickel(II) complex with advanced wave function theory methods should now be spread in the experimental molecular magnetism community, since it may reinforce, if it were still necessary, the interest for new joint theory/experiment works.

\section{Supporting Information Available}

XYZ coordinates of the molecular structure of the $\mathrm{Ni}_{4}$ complex used in this work.

\section{Acknowledgment}

The author thanks N. Guihéry (University of Toulouse, France) for helpful discussions.

\section{References}

(1) Lis, T. Preparation, structure, and magnetic properties of a dodecanuclear mixedvalence manganese carboxylate. Acta Crystallogr. Sect. B 1980, 36, 2042-2046.

(2) Caneschi, A.; Gatteschi, D.; Sessoli, R.; Barra, A. L.; Brunel, L. C.; Guillot, M. Alternating current susceptibility, high field magnetization, and millimeter band EPR evidence for a ground $S=10$ state in $\left[\mathrm{Mn}_{12} \mathrm{O}_{12}\left(\mathrm{CH}_{3} \mathrm{COO}\right)_{16}\left(\mathrm{H}_{2} \mathrm{O}\right)_{4}\right] \cdot 2 \mathrm{CH}_{3} \mathrm{COOH} .4 \mathrm{H}_{2} \mathrm{O}$. J. Am. Chem. Soc. 1991, 113, 5873-5874.

(3) Friedman, J. R.; Sarachik, M. P.; Tejada, J.; Ziolo, R. Macroscopic Measurement of Resonant Magnetization Tunneling in High-Spin Molecules. Phys. Rev. Lett. 1996, 76 , $3830-3833$. 
(4) Thomas, L.; Lionti, F.; Ballou, R.; Gatteschi, D.; Barbara, B. Macroscopic quantum tunnelling of magnetization in a single crystal of nanomagnets. Nature 1996, 383, 145147.

(5) Ruiz, E.; Cirera, J.; Cano, J.; Alvarez, S.; Loose, C.; Kortus, J. Can large magnetic anisotropy and high spin really coexist? Chem. Commun. 2008, 52-54.

(6) Waldmann, O. A Criterion for the Anisotropy Barrier in Single-Molecule Magnets. Inorg. Chem. 2007, 46, 10035-10037.

(7) Ruamps, R.; Maurice, R.; de Graaf, C.; Guihéry, N. Interplay between Local Anisotropies in Binuclear Complexes. Inorg. Chem. 2014, 53, 4508-4516.

(8) Ishikawa, N.; Sugita, M.; Wernsdorfer, W. Quantum Tunneling of Magnetization in Lanthanide Single-Molecule Magnets: Bis(phthalocyaninato)terbium and Bis(phthalocyaninato)dysprosium Anions. Angew. Chem. Int. Ed. 2005, 44, 2931-2935.

(9) Gu, L.; Wu, R. Origins of Slow Magnetic Relaxation in Single-Molecule Magnets. Phys. Rev. Lett. 2020, 125, 117203.

(10) Guo, F.-S.; Day, B. M.; Chen, Y.-C.; Tong, M.-L.; Mansikkamäki, A.; Layfield, R. A. A Dysprosium Metallocene Single-Molecule Magnet Functioning at the Axial Limit. Angew. Chem. Int. Ed. 2017, 56, 11445-11449.

(11) Goodwin, C. A. P.; Ortu, F.; Reta, D.; Chilton, N. F.; Mills, D. P. Molecular magnetic hysteresis at 60 kelvin in dysprosocenium. Nature 2017, 548, 439-442.

(12) Randall McClain, K.; Gould, C. A.; Chakarawet, K.; Teat, S. J.; Groshens, T. J.; Long, J. R.; Harvey, B. G. High-temperature magnetic blocking and magneto-structural correlations in a series of dysprosium(III) metallocenium single-molecule magnets. Chem. Sci. 2018, 9, 8492-8503. 
(13) Guo, F.-S.; Day, B. M.; Chen, Y.-C.; Tong, M.-L.; Mansikkamäki, A.; Layfield, R. A. Magnetic hysteresis up to 80 kelvin in a dysprosium metallocene single-molecule magnet. Science 2018, 362, 1400-1403.

(14) Pederson, M. R.; Khanna, S. N. Magnetic anisotropy barrier for spin tunneling in $\mathrm{Mn}_{12} \mathrm{O}_{12}$ molecules. Phys. Rev. B 1999, 60, 9566-9572.

(15) Baruah, T.; Pederson, M. R. Electronic structure and magnetic anisotropy of the $\left[\mathrm{Co}_{4}(\mathrm{hmp})_{4}\left(\mathrm{CH}_{3} \mathrm{OH}\right)_{4} \mathrm{Cl}_{4}\right]$ molecule. Chem. Phys. Lett. 2002, 360, 144-148.

(16) Postnikov, A. V.; Kortus, J.; Pederson, M. R. Density functional studies of molecular magnets. Phys. Physica Status Solidi 2006, 243, 2533-2572.

(17) Kubica, A.; Kowalewski, J.; Kruk, D.; Odelius, M. Zero-field splitting in nickel(II) complexes: A comparison of DFT and multi-configurational wavefunction calculations. J. Chem. Phys. 2013, 138, 064304.

(18) Escuer, A.; Font-Bardía, M.; Kumar, S. B.; Solans, X.; Vicente, R. Two new nickel(II) cubane compounds derived from pyridine-2-methoxide (Pym): $\mathrm{Ni}_{4}(\mathrm{Pym})_{4} \mathrm{Cl}_{4}\left(\mathrm{CH}_{3} \mathrm{OH}\right)_{4}$ and $\mathrm{Ni}_{4}(\mathrm{Pym})_{4}\left(\mathrm{~N}_{3}\right)_{4}\left(\mathrm{CH}_{3} \mathrm{OH}\right)_{4}$. Crystal structures and magnetic properties. Polyhedron 1999, 18, 909-914.

(19) Moragues-Cánovas, M.; Helliwell, M.; Ricard, L.; Rivière, E.; Wernsdorfer, W.; Brechin, E.; Mallah, T. An $\mathrm{Ni}_{4}$ Single-Molecule Magnet: Synthesis, Structure and Low-Temperature Magnetic Behavior. Eur. J. Inorg. Chem. 2004, 2004, 2219-2222.

(20) Rajaraman, G.; Christensen, K. E.; Larsen, F. K.; Timco, G. A.; Winpenny, R. E. P. Theoretical studies on di- and tetra-nuclear Ni pivalate complexes. Chem. Commun. 2005, 3053-3055.

(21) Herchel, R.; Nemec, I.; Machata, M.; Trávníček, Z. Solvent-induced structural diversity 
in tetranuclear $\mathrm{Ni}(\mathrm{II})$ Schiff-base complexes: the first $\mathrm{Ni}_{4}$ single-molecule magnet with a defective dicubane-like topology. Dalton Trans. 2016, 45, 18622-18634.

(22) Venegas-Yazigi, D.; Cano, J.; Ruiz, E.; Alvarez, S. Theoretical study of the electronic properties and exchange coupling in a $\mathrm{Ni}_{4}$ cubane like single-molecule magnet. Physica B Condens. Matter 2006, 384, 123-125.

(23) Park, K.; Yang, E.-C.; Hendrickson, D. N. Electronic structure and magnetic anisotropy for nickel-based molecular magnets. J. of Appl. Phys. 2005, 97, 10M522.

(24) Cao, C.; Hill, S.; Cheng, H.-P. Strongly Correlated Electrons in the $[\mathrm{Ni}(\mathrm{hmp})(R \mathrm{OH}) \mathrm{X}]_{4}$ Single Molecule Magnet: A DFT + U Study. Phys. Rev. Lett. 2008, 100, 167206.

(25) Maurice, R.; de Graaf, C.; Guihéry, N. Theoretical determination of spin Hamiltonians with isotropic and anisotropic magnetic interactions in transition metal and lanthanide complexes. Phys. Chem. Chem. Phys. 2013, 15, 18784-18804.

(26) Gomez-Coca, S.; Cremades, E.; Aliaga-Alcalde, N.; Ruiz, E. Mononuclear SingleMolecule Magnets: Tailoring the Magnetic Anisotropy of First-Row Transition-Metal Complexes. J. Am. Chem. Soc. 2013, 135, 7010-7018.

(27) Atanasov, M.; Aravena, D.; Suturina, E.; Bill, E.; Maganas, D.; Neese, F. First principles approach to the electronic structure, magnetic anisotropy and spin relaxation in mononuclear 3d-transition metal single molecule magnets. Coord. Chem. Rev. 2015, 289-290, $177-214$.

(28) Gómez-Coca, S.; Aravena, D.; Morales, R.; Ruiz, E. Large magnetic anisotropy in mononuclear metal complexes. Coord. Chem. Rev. 2015, 289-290, 379-392.

(29) Maurice, R.; Broer, R.; Guihéry, N.; de Graaf, C. 2017; Zero-field splitting in transition metal complexes: $A b$ initio calculations, effective Hamiltonians, model Hamiltonians, 
and crystal-field models. In Handbook of Relativistic Quantum Chemistry, Ed. W. Liu, Springer-Verlag Berlin Heidelberg, 765-796 (2017).

(30) Maurice, R.; Bastardis, R.; Graaf, C. d.; Suaud, N.; Mallah, T.; Guihéry, N. Universal Theoretical Approach to Extract Anisotropic Spin Hamiltonians. J. Chem. Theory Comput. 2009, 5, 2977-2984.

(31) Maurice, R.; Vendier, L.; Costes, J.-P. Magnetic Anisotropy in $\mathrm{Ni}^{\mathrm{II}}-\mathrm{Y}^{\mathrm{III}}$ Binuclear Complexes: On the Importance of Both the First Coordination Sphere of the $\mathrm{Ni}^{\mathrm{II}}$ Ion and the $\mathrm{Y}^{\mathrm{III}}$ Ion Belonging to the Second Coordination Sphere. Inorg. Chem. 2011, $\underline{50,}, 11075-11081$.

(32) Costes, J.-P.; Maurice, R.; Vendier, L. Pentacoordinate Ni ${ }^{\mathrm{II}}$ Complexes: Preparation, Magnetic Measurements, and ab initio Calculations of the Magnetic Anisotropy Terms. Chem. Eur. J. 2012, 18, 4031-4040.

(33) Chibotaru, L. F.; Ungur, L. Ab initio calculation of anisotropic magnetic properties of complexes. I. Unique definition of pseudospin Hamiltonians and their derivation. J. Chem. Phys. 2012, 137, 064112.

(34) Ruamps, R.; Batchelor, L. J.; Maurice, R.; Gogoi, N.; Jiménez-Lozano, P.; Guihéry, N.; de Graaf, C.; Barra, A.-L.; Sutter, J.-P.; Mallah, T. Origin of the Magnetic Anisotropy in Heptacoordinate $\mathrm{Ni}^{\mathrm{II}}$ and $\mathrm{Co}^{\mathrm{II}}$ Complexes. Chem. Eur. J. 2013, 19, 950-956.

(35) Ruamps, R.; Maurice, R.; Batchelor, L.; Boggio-Pasqua, M.; Guillot, R.; Barra, A. L.; Liu, J.; Bendeif, E.-E.; Pillet, S.; Hill, S. et al. Giant Ising-Type Magnetic Anisotropy in Trigonal Bipyramidal Ni(II) Complexes: Experiment and Theory. J. Am. Chem. Soc. 2013, 135, 3017-3026.

(36) Gómez-Coca, S.; Cremades, E.; Aliaga-Alcalde, N.; Ruiz, E. Huge Magnetic Anisotropy in a Trigonal-Pyramidal Nickel(II) Complex. Inorg. Chem. 2014, 53, 676-678. 
(37) Singh, S. K.; Gupta, T.; Badkur, P.; Rajaraman, G. Magnetic Anisotropy of Mononuclear $\mathrm{Ni}^{\mathrm{II}}$ Complexes: On the Importance of Structural Diversity and the Structural Distortions. Chem. Eur. J. 2014, 20, 10305-10313.

(38) Jiang, S.-D.; Maganas, D.; Levesanos, N.; Ferentinos, E.; Haas, S.; Thirunavukkuarasu, K.; Krzystek, J.; Dressel, M.; Bogani, L.; Neese, F. et al. Direct Observation of Very Large Zero-Field Splitting in a Tetrahedral $\mathrm{Ni}^{\mathrm{II}} \mathrm{Se}_{4}$ Coordination Complex. J. Am. Chem. Soc. 2015, 137, 12923-12928.

(39) Cahier, B.; Perfetti, M.; Zakhia, G.; Naoufal, D.; El-Khatib, F.; Guillot, R.; Rivière, E.; Sessoli, R.; Barra, A.-L.; Guihéry, N. et al. Magnetic Anisotropy in Pentacoordinate $\mathrm{Ni}^{\mathrm{II}}$ and $\mathrm{Co}^{\mathrm{II}}$ Complexes: Unraveling Electronic and Geometrical Contributions. Chem. Eur. J. 2017, 23, 3648-3657.

(40) Costes, J.-P.; Mallet-Ladeira, S.; Vendier, L.; Maurice, R.; Wernsdorfer, W. Influence of Ancillary Ligands and Solvents on the Nuclearity of Ni-Ln complexes. Dalton Trans. 2019, 48, 3404-3414.

(41) Suaud, N.; Rogez, G.; Rebilly, J.-N.; Bouammali, M.-A.; Guihéry, N.; Barra, A.-L.; Mallah, T. Playing with Magnetic Anisotropy in Hexacoordinated Mononuclear Ni(II) Complexes, An Interplay Between Symmetry and Geometry. Appl. Magn. Reson. 2020, $\underline{51}, 1215-1231$.

(42) Maurice, R.; Guihéry, N.; Bastardis, R.; de Graaf, C. Rigorous Extraction of the Anisotropic Multispin Hamiltonian in Bimetallic Complexes from the Exact Electronic Hamiltonian. J. Chem. Theory Comput. 2010, $\underline{6}, 55-65$.

(43) Maurice, R.; de Graaf, C.; Guihéry, N. Magnetic anisotropy in binuclear complexes in the weak-exchange limit: From the multispin to the giant-spin Hamiltonian. Phys. Rev. B 2010, 81, 214427. 
(44) Herchel, R.; Boča, R.; Krzystek, J.; Ozarowski, A.; Durán, M.; van Slageren, J. Definitive Determination of Zero-Field Splitting and Exchange Interactions in a Ni(II) Dimer: Investigation of $\left[\mathrm{Ni}_{2}(\mathrm{en})_{4} \mathrm{Cl}_{2}\right] \mathrm{Cl}_{2}$ Using Magnetization and Tunable-Frequency HighField Electron Paramagnetic Resonance. J. Am. Chem. Soc. 2007, 129, 10306-10307.

(45) Palmer, D. C. Visualization and analysis of crystal structures using CrystalMaker software. Z. Kristall. 2015, 230, 559-572.

(46) Petit, S.; Pilet, G.; Luneau, D.; Chibotaru, L. F.; Ungur, L. A dinuclear cobalt(II) complex of calix[8]arenes exibiting strong magnetic anisotropy. Dalton Trans. 2007, $4582-4588$.

(47) Heisenberg, W. Zur Theorie des Ferromagnetismus. Z. Phys. 1928, 49, 619-636.

(48) Dirac, P. A. M. Quantum mechanics of many-electron systems. Proc. R. Soc. A 1929, 123, 714-733.

(49) Van Vleck, J. H. A Survey of the Theory of Ferromagnetism. Rev. Mod. Phys. 1945, $\underline{17}, 27-47$.

(50) Moreira, I. d. P. R.; Illas, F. A unified view of the theoretical description of magnetic coupling in molecular chemistry and solid state physics. Phys. Chem. Chem. Phys. 2006, $\underline{8}, 1645-1659$.

(51) Moreira, I. d. P. R.; Suaud, N.; Guihéry, N.; Malrieu, J. P.; Caballol, R.; Bofill, J. M.; Illas, F. Derivation of spin Hamiltonians from the exact Hamiltonian: Application to systems with two unpaired electrons per magnetic site. Phys. Rev. B 2002, 66, 134430.

(52) Bastardis, R.; Guihéry, N.; de Graaf, C. Microscopic origin of isotropic non-Heisenberg behavior in $S=1$ magnetic systems. Phys. Rev. B 2007, $\underline{76}, 132412$.

(53) Bastardis, R.; Guihéry, N.; de Graaf, C. Isotropic non-Heisenberg terms in the magnetic coupling of transition metal complexes. J. Chem. Phys. 2008, 129, 104102. 
(54) Bencini, A.; Totti, F. On the importance of the biquadratic terms in exchange coupled systems: A post-HF investigation. Inorg. Chim. Acta 2008, 361, 4153-4156.

(55) Queralt, N.; Taratiel, D.; de Graaf, C.; Caballol, R.; Cimiraglia, R.; Angeli, C. On the applicability of multireference second-order perturbation theory to study weak magnetic coupling in molecular complexes. J. Comput. Chem. 2008, 29, 994-1003.

(56) Spivak, M.; Angeli, C.; Calzado, C. J.; de Graaf, C. Improving the calculation of magnetic coupling constants in MRPT methods. J. Comp. Chem. 2014, 35, 1665-1671.

(57) Roemelt, M.; Krewald, V.; Pantazis, D. A. Exchange Coupling Interactions from the Density Matrix Renormalization Group and N-Electron Valence Perturbation Theory: Application to a Biomimetic Mixed-Valence Manganese Complex. J. Chem. Theory Comput. 2018, 14, 166-179.

(58) Calzado, C. J.; Cabrero, J.; Malrieu, J.-P.; Caballol, R. Analysis of the magnetic coupling in binuclear complexes. I. Physics of the coupling. J. Chem. Phys. 2002, 116, $2728-2747$.

(59) Calzado, C. J.; Cabrero, J.; Malrieu, J.-P.; Caballol, R. Analysis of the magnetic coupling in binuclear complexes. II. Derivation of valence effective Hamiltonians from $a b$ initio CI and DFT calculations. J. Chem. Phys. 2002, 116, 3985-4000.

(60) Calzado, C. J.; Angeli, C.; Taratiel, D.; Caballol, R.; Malrieu, J.-P. Analysis of the magnetic coupling in binuclear systems. III. The role of the ligand to metal charge transfer excitations revisited. J. Chem. Phys. 2009, 131, 044327.

(61) Malrieu, J.-P.; Caballol, R.; Calzado, C. J.; de Graaf, C.; Guihéry, N. Magnetic Interactions in Molecules and Highly Correlated Materials: Physical Content, Analytical Derivation, and Rigorous Extraction of Magnetic Hamiltonians. Chem. Rev. 2014, 114, 429-492. 
(62) Boča, R. Theoretical foundations of molecular magnetism; Elsevier, Amsterdam, 1999.

(63) Maurice, R.; Verma, P.; Zadrozny, J. M.; Luo, S.; Borycz, J.; Long, J. R.; Truhlar, D. G.; Gagliardi, L. Single-Ion Magnetic Anisotropy and Isotropic Magnetic Couplings in the Metal-Organic Framework $\mathrm{Fe}_{2}$ (dobdc). Inorg. Chem. 2013, 52, 9379-9389.

(64) Verma, P.; Maurice, R.; Truhlar, D. G. Adsorbate-Induced Changes in Magnetic Interactions in $\mathrm{Fe}_{2}$ (dobdc) with Adsorbed Hydrocarbon Molecules. J. Phys. Chem. C 2016, 120, 9933-9948.

(65) Barandiarán, Z.; Seijo, L. The ab initio model potential representation of the crystalline environment. Theoretical study of the local distortion on $\mathrm{NaCl}: \mathrm{Cu}^{+}$. J. Chem. Phys. 1988, 89, 5739-5746.

(66) Retegan, M.; Cox, N.; Pantazis, D. A.; Neese, F. A First-Principles Approach to the Calculation of the on-Site Zero-Field Splitting in Polynuclear Transition Metal Complexes. Inorg. Chem. 2014, 53, 11785-11793.

(67) Kahn, O. Molecular magnetism; VCH, New York, 1993.

(68) Abragam, A.; Bleaney, B. Electron paramagnetic resonance of transition ions; Dover Publications, New York, 1986.

(69) Maurice, R.; de Graaf, C.; Guihéry, N. Magnetostructural relations from a combined ab initio and ligand field analysis for the nonintuitive zero-field splitting in $\mathrm{Mn}$ (III) complexes. J. Chem. Phys. 2010, 133, 084307.

(70) Stevens, K. W. H. Matrix Elements and Operator Equivalents Connected with the Magnetic Properties of Rare Earth Ions. Proc. Phys. Soc. A 1952, 65, 209-215.

(71) Altshuler, S. A.; Kozyrev, B. M. Electron paramagnetic resonance of transition ions; 1974; Electron paramagnetic resonance in compounds of transition elements, HalstedWiley, New York, New York. 
(72) Rudowicz, C.; Chung, C. Y. The generalization of the extended Stevens operators to higher ranks and spins, and a systematic review of the tables of the tensor operators and their matrix elements. J. Phys.: Condens. Matter 2004, 16, 5825-5847.

(73) Douglas, M.; Kroll, N. M. Quantum electrodynamical corrections to the fine structure

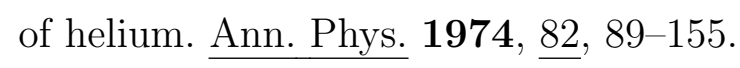

(74) Hess, B. A. Relativistic electronic-structure calculations employing a two-component no-pair formalism with external-field projection operators. Phys. Rev. A 1986, $\underline{33}$, $3742-3748$.

(75) Jansen, G.; Hess, B. A. Revision of the Douglas-Kroll transformation. Phys. Rev. A 1989, 39, 6016-6017.

(76) Roos, B. O.; Taylor, P. R.; Siegbahn, P. E. A complete active space SCF method (CASSCF) using a density matrix formulated super-CI approach. Chem. Phys. 1980, $\underline{48}, 157-173$.

(77) Roos, B. O. In Theory and applications of computational chemistry: The first forty years, Eds. Dykstra, C. E., Frenking, G., Kim, K. S., Scuseria, G. E., Elsevier Amsterdam, 725-764 (2005).

(78) Angeli, C.; Cimiraglia, R.; Evangelisti, S.; Leininger, T.; Malrieu, J.-P. Introduction of $n$-electron valence states for multireference perturbation theory. J. Chem. Phys. 2001, $\underline{114}, 10252-10264$.

(79) Angeli, C.; Cimiraglia, R.; Malrieu, J.-P. $N$-electron valence state perturbation theory: a fast implementation of the strongly contracted variant. Chem. Phys. Lett. 2001, 350, 297-305.

(80) Angeli, C.; Cimiraglia, R.; Malrieu, J.-P. $N$-electron valence state perturbation theory: 
A spinless formulation and an efficient implementation of the strongly contracted and of the partially contracted variants. J. Chem. Phys. 2002, 117, 9138-9153.

(81) Teichteil, C.; Pélissier, M.; Spiegelmann, F. Ab initio molecular calculations including spin-orbit coupling. I. Method and atomic tests. Chem. Phys. 1983, 81, 273-282.

(82) Llusar, R.; Casarrubios, M.; Barandiarán, Z.; Seijo, L. Ab initio model potential calculations on the electronic spectrum of $\mathrm{Ni}^{2+}$-doped $\mathrm{MgO}$ including correlation, spin-orbit and embedding effects. J. Chem. Phys. 1996, 105, 5321-5330.

(83) F. Neese. ORCA - An ab initio, DFT and semiempirical SCF-MO package, Max-PlanckInstitut für Bioanorganische Chemie, Mülheim an der Ruhr.

(84) Neese, F. Efficient and accurate approximations to the molecular spin-orbit coupling operator and their use in molecular $g$-tensor calculations. J. Chem. Phys. 2005, $\underline{122}$, 034107 .

(85) Weigend, F.; Ahlrichs, R. Balanced basis sets of split valence, triple zeta valence and quadruple zeta valence quality for H to Rn: Design and assessment of accuracy. Phys. Chem. Chem. Phys. 2005, 7, 3297-3305.

(86) Hellweg, A.; Hättic, C.; Höfener, S.; Klopper, W. Optimized accurate auxiliary basis sets for RI-MP2 and RI-CC2 calculations for the atoms Rb to Rn. Theor. Chem. Acc. 2007, 117, 587-597.

(87) Bloch, C. Sur la théorie des perturbations des états liés. Nucl. Phys. 1958, 6, 329-347.

(88) des Cloizeaux, J. Extension d'une formule de Lagrange à des problèmes de valeurs propres. Nucl. Phys. 1960, 20, 321-346.

(89) Löwdin, P.-O. In On the Nonorthogonality Problem; Löwdin, P.-O., Ed.; Advances in Quantum Chemistry; Academic Press, 1970; Vol. 5; pp 185-199. 
(90) Bouchafra, Y.; Shee, A.; Réal, F.; Vallet, V.; Severo Pereira Gomes, A. Predictive Simulations of Ionization Energies of Solvated Halide Ions with Relativistic Embedded Equation of Motion Coupled Cluster Theory. Phys. Rev. Lett. 2018, 121, 266001.

(91) Ostrovsky, S. M.; Werner, R.; Brown, D. A.; Haase, W. Magnetic properties of dinuclear cobalt complexes. Chem. Phys. Lett. 2002, 353, 290-294.

(92) Yang, E.-C.; Hendrickson, D. N.; Wernsdorfer, W.; Nakano, M.; Zakharov, L. N.; Sommer, R. D.; Rheingold, A. L.; Ledezma-Gairaud, M.; Christou, G. Cobalt singlemolecule magnet. J. Appl. Phys. 2002, 91, 7382-7384.

(93) Atanasov, M.; Ganyushin, D.; Pantazis, D. A.; Sivalingam, K.; Neese, F. Detailed Ab Initio First-Principles Study of the Magnetic Anisotropy in a Family of Trigonal Pyramidal Iron(II) Pyrrolide Complexes. Inorg. Chem. 2011, 50, 7460-7477. 


\section{For Table of Contents Only:}

\section{TOC graphic:}

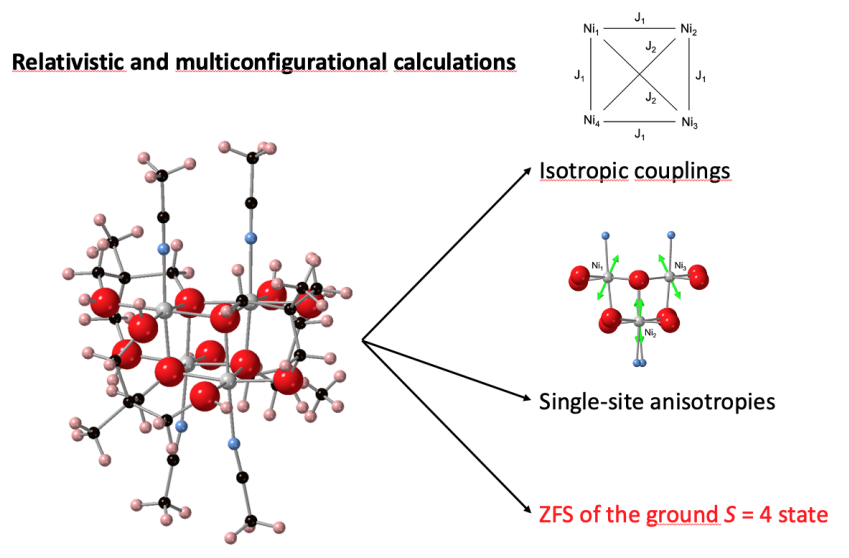

\section{Synopsis:}

The isotropic couplings, the single-site anisotropies and the ZFS of the ground $S=4$ state of a cubane-like $\mathrm{Ni}_{4}$ complex are computed by means of state-of-the-art relativistic and multiconfigurational calculations. Weak ferromagnetic couplings are confirmed between the nickel(II) sites, together with a weak uniaxial anisotropy for the coupled $S=4$ state. The small value of $D$ is explained by the actual mismatch between the single-site anisotropy axes. 